\title{
Real time current profile control at JET
}

\author{
J.A. Romero ${ }^{\text {a,* }}$, J.M. de la Cruz ${ }^{\text {b }, ~ T . ~ D o b b i n g ~}{ }^{\text {a }}$, J. Ellis ${ }^{\text {a }}$, B. Fisher ${ }^{\text {a }}$, Q.A. King ${ }^{\text {a }}$, \\ F. Söldner ${ }^{\text {a }}$, N. Zornig a \\ a JET Joint Undertaking, Abingdon, Oxon OX14 3EA, UK \\ ${ }^{\mathrm{b}}$ Universidad Complutense de Madrid, Avda Complutense $S / N$, Madrid, Spain
}

Received 30 September 1997; received in revised form 5 February 1998

\begin{abstract}
A plasma current profile control system has been designed and tested at Joint European Torus (JET). The system uses the one turn loop voltage for lower hybrid current drive (LHCD) control, and the normalised second current moment of the plasma current distribution for off-axis current control. These provide relatively simple measurements and calculations suitable for real time operation. Models for the response of the loop voltage and second current moment to Lower Hybrid power input are derived and validated against JET data. Based on these models, the components of the second current moment and loop voltage control systems are designed to suit prescribed performance. Loop voltage has been controlled in $2.5 \mathrm{MA}$ discharges at $66 \%$ reduction, providing the means for LHCD regulation. Second current moment control during the current rise phase has contributed to $60 \%$ improvement of the neutron yield performance of shear optimisation discharges at JET. (C) 1998 Elsevier Science S.A. All rights reserved.
\end{abstract}

Keywords: Current profile control; Lower hybrid current drive control; Magneto hydro dynamic

\section{Introduction}

The shape of the plasma current profile is one of the most important factors determining the plasma magneto hydro dynamic (MHD) stability and confinement in Tokamaks. For this reason, plasma current profile control has received considerable attention in recent years.

One of the tools for plasma current profile control at Joint European Torus (JET) [1] is the lower hybrid current drive system (LHCD) [2,3].

\footnotetext{
* Corresponding author.
}

Current profile control experiments with lower hybrid waves at JET are usually conducted in open loop, by careful pre-programming of the LHCD heating power waveforms. However, this type of operation is affected considerably by changing properties of the plasma, and the desired target current profiles are not always obtained.

Feedback control tools and techniques provide a solution to this problem [4]. This paper describes a current profile feedback control system that has been developed for the LHCD system at JET which provides the capability for steady state scenarios, and eliminates the need for re-adjustments on the power waveforms. With this control 


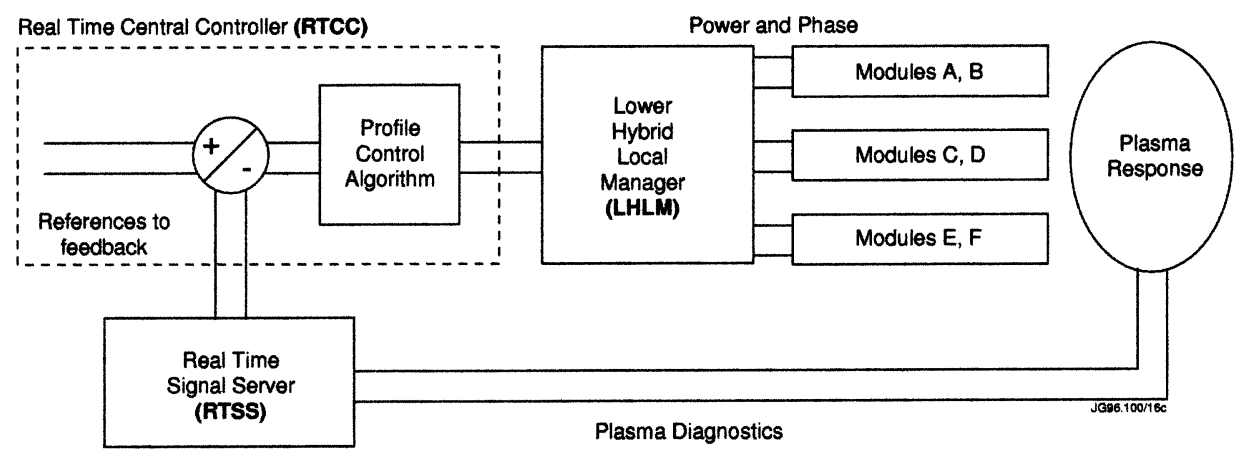

Fig. 1. Schematics of the current profile control system for the LHCD system at JET. The diagnostics for feedback are currently the loop voltage and the normalised second current moment.

system, it is now possible to control the non-inductive current generated by the LHCD system and the relative peaking of the current profile.

The control system uses the loop voltage for non-inductive control, and a dimensionless quantity derived from the second moment of the current profile for off-axis current control.

The philosophy of the control system design has been to develop the simplest possible mathematical model for the plasma response, namely a non-linear state space model [5] for the loop voltage, and a transfer function [5] model for the second current moment. These models will be presented and validated against data from JET discharges.

This initial development has improved the operation of current profile control experiments with lower hybrid, and it has contributed to an improvement on fusion yield performance in shear optimization experiments at JET. This illustrates the potential benefits of current profile feedback control systems for future devices such as ITER, where confinement and stability are crucial.

\section{General description of the system}

The control system has been implemented in a front end computer working with sample data known at JET as the real time power control system (RTPC) [6]. The control system consists of three main parts (Fig. 1):
(1) The real time signal server (RTSS) collects a signal from numerous plasma diagnostics and performs simple processing such as calibration and filtering.

(2) The real time central controller (RTCC) reads from a common memory area, shared with RTSS, the relevant diagnostics selected for feedback. It then executes a network of interconnected algorithms prescribed by the user. These are typically waveform generation of requested feedforward powers, references for feedback, feedback timing, filtering and proportional integral derivative (PID) control algorithms. The network output is calculated every $10 \mathrm{~ms}$. The profile control algorithm is implemented in this area. The result of the processing is a power or phase demand sent through an Ethernet connection to the lower hybrid local manager (LHLM).

(3) The LHLM reads the power and phase requested by the RTCC and performs local feedback control of the power generated by the $\mathrm{LH}$ plant. Independent control of power and phase is possible for the 6 power modules that constitute the plant [3].

Initially, while establishing the engineering basis of this new control concept, loop voltage and second current moment feedback loops have been implemented. Future current profile diagnostic developments at JET may allow control of the current profile in the central region of the plasma, such as the central value of the safety factor $\left(q_{0}\right)$ $[7,8]$. 


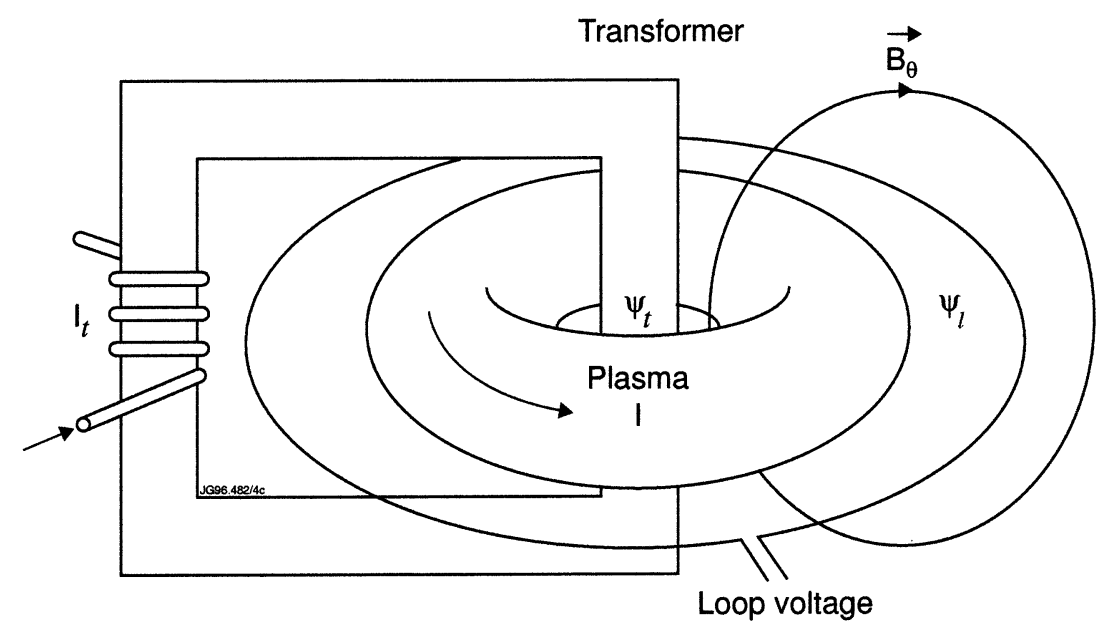

Fig. 2. Simplified schematic of a Tokamak and the loop voltage measurement.

\section{Lower hybrid current drive control}

Tokamak operation is limited by the amount of magnetic flux available in the transformer primary. To allow continuous operation, there are several non inductive current drive methods. One of the most interesting ones is the LHCD. The LHCD system at JET generates microwave power at $3.7 \mathrm{GHz}$. This system has achieved full current drive in $X$-point configurations in the range $0.7-3$ MA and line average electron density, $\bar{n}_{\mathrm{e}}$, in the range $0.7-2.0 \times 10^{19} \mathrm{~m}^{-3}$, with current drive efficiency, $\eta_{\mathrm{CD}}$, increasing with electron temperature up to $0.3 \times 10^{20} \mathrm{AW}^{-1} \mathrm{~m}^{-2}$. However, in some cases, variations in the plasma conditions may lead to uncontrolled excursions of the non-inductive current generated by the LH system. In order to obtain steady state scenarios, a non-inductive current control system with LH waves has been developed and tested. This section presents the design of the LHCD control system and the experimental results.

\subsection{Loop voltage}

The loop voltage is the voltage created in a circular loop concentric with the plasma column as a result of the variation of the poloidal magnetic flux linked through it (Fig. 2). By extension of this concept, the loop voltage in an arbitrary flux surface is minus the time derivative of the poloidal magnetic flux relative to that surface. At JET, a real time system [9] computes the poloidal flux function outside the plasma every $2 \mathrm{~ms}$. This gives the loop voltage at the plasma boundary, where it is otherwise impossible to obtain a direct measurement. There are two more loops taking direct measurements of the loop voltage in real time; one placed slightly above the mid-plane of the plasma and inside the vacuum vessel, and another placed below the plasma and outside the vacuum vessel. By simple inspection of Fig. 2, it is clear that any change in the magnetic flux through the loop will generate a voltage, according to Lenz's law. The change in the flux can be produced by a variation in the plasma current or primary transformer flux. Plasma and transformer circuits are inductively coupled, and for this reason the loop voltage measurement contains mixed information regarding non-inductive current drive, plasma resistance and plasma inductance changes. In order to obtain exact expressions for the Tokamak equivalent parameters and its relationship with the loop voltage, the Tokamak equivalent circuit is presented in the Section 3.2.

\subsection{Tokamak equivalent circuit}

A Tokamak can be described as a distributedparameter electric network (Fig. 3). Energy stor- 
age in the poloidal magnetic field is accounted for by a series inductance $L$. Ohmic power loss in the plasma is taken into account by a series resistance $R$. Total plasma current is represented by $I$. Non inductive current drive can be described by a parallel current source $\hat{I}$ or a series voltage supply $\hat{V}=R \hat{I}$. The coupling between the plasma and the loop voltage is accounted for by a mutual inductance $M$. The primary circuit of the transformer is described by a voltage $V_{\mathrm{t}}$, a resistance $R_{\mathrm{t}}$ and an inductance $L_{\mathrm{t}}$. The mutual inductance $M_{\mathrm{t}}$ describes the coupling between transformer primary and plasma, and is considered the same as the mutual inductance between transformer and loop voltage. It is assumed that the loop voltage $V$ is measured along a poloidal flux surface, and its relationship with the distributed parameters of Fig. 3(a) can be described by

$$
V=R I-\hat{V}+\frac{\mathrm{d}}{\mathrm{d} t}((L-M) I)
$$

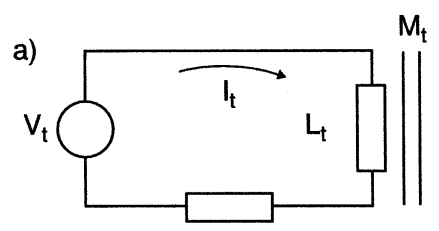

$R_{\mathrm{t}}$
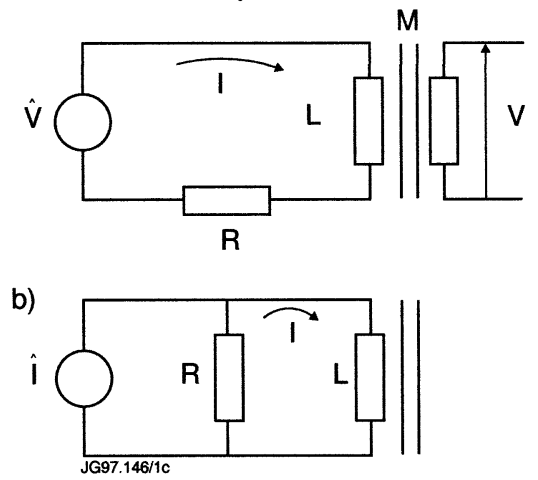

Fig. 3. Tokamak equivalent circuit with non-inductive current drive. The plasma is represented by a resistance $R$ and an inductance $L$. The mutual inductance between the plasma and measuring loop is $M$. The mutual inductance between the transformer primary and the plasma or the loop is $M_{\mathrm{t}}$. The primary circuit of the transformer is described by a voltage $V_{\mathrm{t}}$, a resistance $R_{\mathrm{t}}$ and an inductance $L_{\mathrm{t}}$. The non-inductive current effect is represented by: (a) an ideal voltage power supply $V$; and (b) an ideal current source $\hat{I}$.
On the other hand, a Poynting's theorem analysis for the poloidal magnetic field $B_{\theta}$ in toroidal geometry leads to the energy balance equation

$\frac{1}{2 \mu_{0}} \frac{\mathrm{d}}{\mathrm{d} t} \int_{\Omega} B_{\theta}^{2} \mathrm{~d} V+\int_{\Omega} \eta j_{\phi}^{2} \mathrm{~d} V-\int_{\Omega} \eta j_{\phi} \hat{j_{\phi}} \mathrm{d} V=V I$

where $\mu_{0}$ is the vacuum magnetic permeability, $j_{\phi}$ and $\hat{j}_{\phi}$ are the total and non-inductive plasma current profiles in the toroidal direction and $\eta$ is the plasma parallel resistivity profile. In obtaining this expression, the toroidal electric field $E_{\phi}$ was written as a function of the non inductive current profile $\hat{j_{\phi}}$, using Ohm's law

$E_{\phi}=\eta\left(j_{\phi}-\hat{j_{\phi}}\right)$

Comparing Eqs. (1) and (2), we obtain

$R=\frac{\int_{\Omega} \eta j_{\phi}^{2} \mathrm{~d} V}{I^{2}}$

$\hat{V}=\frac{\int_{\Omega} \eta j_{\phi} \hat{j}_{\phi} \mathrm{d} V}{I}$

$I \frac{\mathrm{d}}{\mathrm{d} t}((L-M) I)=\frac{1}{2} \mu_{0} \frac{\mathrm{d}}{\mathrm{d} t} \int_{\Omega} B_{\theta}^{2} \mathrm{~d} V$

where the integration volume $\Omega$ is limited by the flux surface that intersects with the voltage loop. The volume $\Omega$ can be split into two regions $\Omega_{\mathrm{i}}$ and $\Omega_{\mathrm{e}}$ which are internal and external to the plasma. The internal region $\Omega_{\mathrm{i}}$ is limited by the last closed flux surface (LCFS). The external region $\Omega_{\mathrm{e}}$ is limited externally by the flux surface that intersects the measuring loop and internally by the LCFS.

When Eq. (6) is integrated in the interval $(0, t)$, the inductive element $L-M$ can be written explicitly as a function of the internal $l_{\mathrm{i}}$ and external $l_{\mathrm{e}}$ inductances,

$$
\begin{aligned}
& L-M=\mu_{0} \frac{r_{0}}{2}\left(l_{\mathrm{i}}+l_{\mathrm{e}}+l_{0}\right), \\
& l_{\mathrm{i}}=\frac{1}{\mu_{0}^{2} I^{2} r_{0}} \int_{\Omega_{\mathrm{i}}} B_{\theta}^{2} \mathrm{~d} V \\
& l_{\mathrm{e}}=\frac{1}{\mu_{0}^{2} I^{2} r_{0}} \int_{\Omega_{\mathrm{e}}} B_{\theta}^{2} \mathrm{~d} V
\end{aligned}
$$



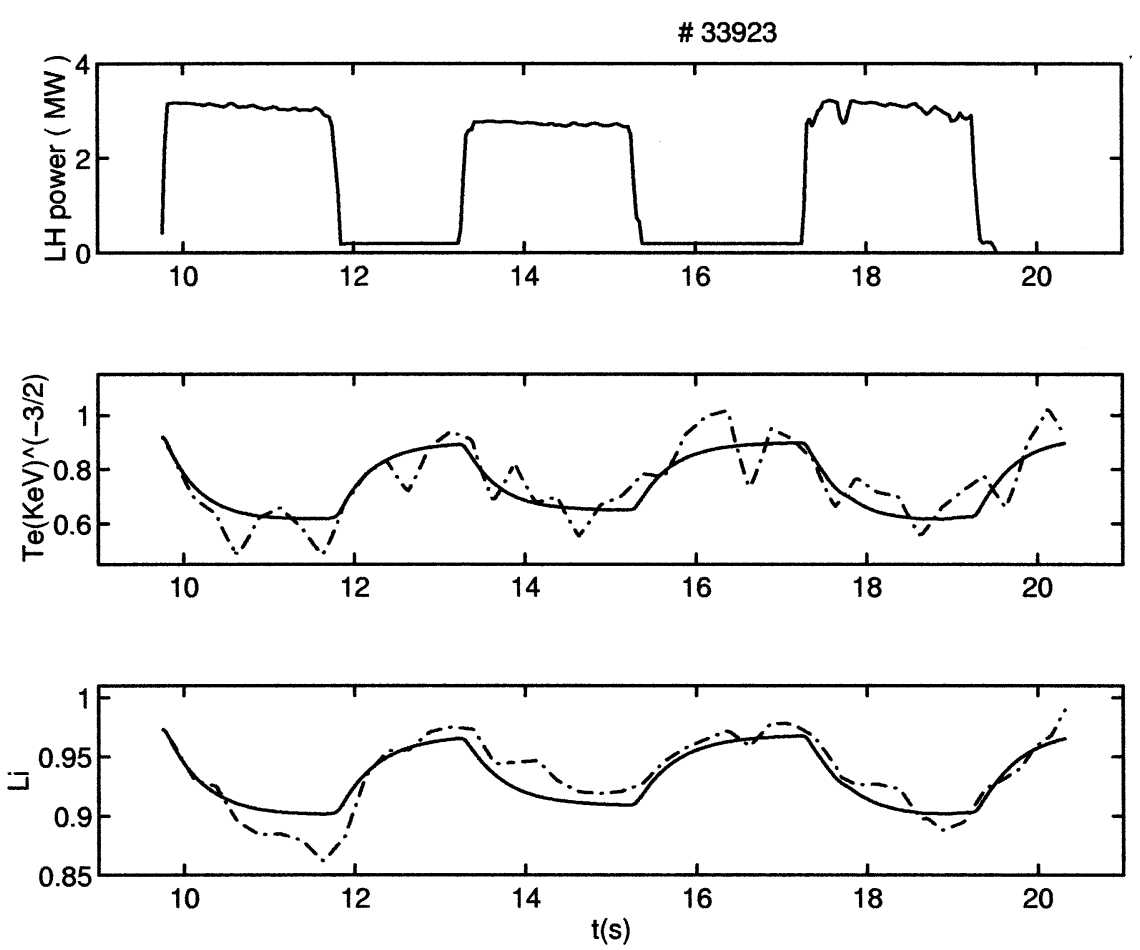

Fig. 4. Loop voltage model internal states vs. experimental data. Top: Lower hybrid power. Middle: First state simulation (solid) compared with actual values (dot-dash). Bottom: Internal inductance simulation (solid) compared with actual values (dot-dash).

$l_{0}=\frac{1}{I} \int_{0}^{t}\left(l_{\mathrm{i}}+l_{\mathrm{e}}\right) \frac{\mathrm{d} I}{\mathrm{~d} t} \mathrm{~d} t \simeq \frac{1}{I} \int_{0}^{t_{\mathrm{r}}}\left(l_{\mathrm{i}}+l_{\mathrm{e}}\right) \frac{\mathrm{d} I}{\mathrm{~d} t} \mathrm{~d} t$

where the major plasma radius is represented by $r_{0}$ to avoid confusion with the plasma resistance $R$. The time integrated inductance $l_{0}$ is constant after the initial current rise phase $\left(0, t_{\mathrm{r}}\right)$, since the plasma current is usually kept constant during the steady state phase of the discharge.

It must be emphasized that Eqs. (4) and (5) and Eq. (7) are the only definitions for the Tokamak parameters that are consistent with loop voltage measurements.

The internal inductance can also be written as a function of the fluxes at the plasma boundary $\psi_{\mathrm{b}}$ and plasma centre $\psi_{0}$

$l_{\mathrm{i}}=\frac{2 \pi\left(\psi_{0}-\psi_{\mathrm{b}}\right)}{\mu_{0} r_{0} I}\left(1-\frac{\int_{\Omega_{\mathrm{i}}} \psi_{\mathrm{n}} j_{\phi} \mathrm{d} A}{I}\right)$,

where $\psi_{\mathrm{n}}=\frac{\left(\psi-\psi_{0}\right)}{\left(\psi_{\mathrm{b}}-\psi_{0}\right)}$

is the normalised flux function and $\mathrm{d} A$ is a differential element of plasma cross-section. In obtaining Eq. (8), we have made use of the identity

$\int B_{\theta}^{2} \mathrm{~d} V=\oint\left(\mathbf{A}_{\phi} \times \mathbf{B}_{\theta}\right) \cdot \mathrm{d} S+\mu_{0} \int \mathbf{A}_{\phi} \cdot \mathbf{j}_{\phi} \mathrm{d} V$

together with the choice for the potential vector

$\mathbf{A}_{\phi}=\frac{\psi-\psi_{0}}{r} \mathbf{u}_{\phi}$

where $\boldsymbol{u}_{\phi}$ is a unitary vector in the toroidal direction.

Similarly, the external inductance can be written as a function of the flux at the loop location $\psi_{\mathrm{i}}$ and at the plasma boundary $\psi_{\mathrm{b}}$,

$l_{\mathrm{e}}=\frac{2 \pi\left(\psi_{\mathrm{b}}-\psi_{l}\right)}{\mu_{0} r_{0} I}$ 


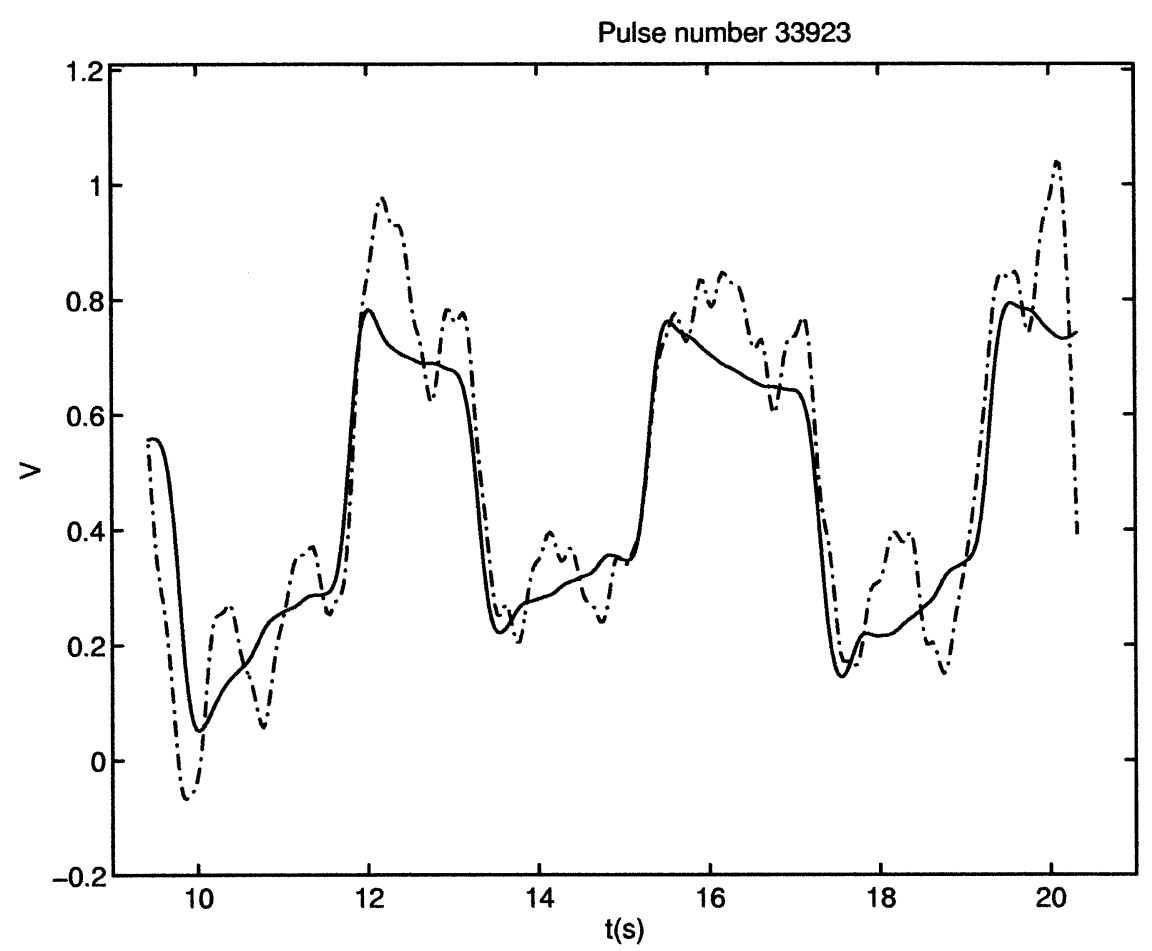

Fig. 5. Comparison of the boundary loop voltage (dot-dash) and the loop voltage model output (solid).

Using the new definitions given by Eq. (7), Eq. (1) can be written as

$$
V=R I-\hat{V}+\mu_{0} \frac{r_{0}}{2} \frac{\mathrm{d}}{\mathrm{d} t}\left(l_{\mathrm{i}}+l_{\mathrm{e}}\right)+\mu_{0} r_{0}\left(l_{\mathrm{i}}+l_{\mathrm{e}}\right) \frac{\mathrm{d} I}{\mathrm{~d} t}
$$

When the loop voltage measurement in Eq. (1) is calculated at the LCFS, $l_{\mathrm{e}}=0$, and Eq. (1) is written as

$$
V_{\mathrm{b}}=R I-\hat{V}+\mu_{0} \frac{r_{0}}{2} I \frac{\mathrm{d} l_{\mathrm{i}}}{\mathrm{d} t}+\mu_{0} r_{0} l_{\mathrm{i}} \frac{\mathrm{d} I}{\mathrm{~d} t}
$$

where in this case, $V_{\mathrm{b}}$ is the loop voltage at the plasma boundary. Due to there being no currents in the region external to the plasma, the parameters $R$ and $\hat{V}$ are still given by the Eqs. (4) and (5).

If the measuring loop is placed just below the plasma, the external inductance depends solely on geometrical factors and is given by

$l_{\mathrm{e}}=\ln \frac{b}{a}$ where $a$ is a characteristic dimension for the minor plasma radius and $b$ is the distance between the plasma current center $\left(r_{0}, z_{0}\right)$ and the measuring loop. For the JET parameters $a=1.2, b=2.3$, we obtain $l_{\mathrm{e}} \cong 0.65$. As long as the plasma shape and the position are kept constant, the external inductance can be considered constant, and the loop measurement Eq. (1) is reduced again to Eq. (14).

Alternatively, as shown in Fig. 3(b), the effect of the non-inductive current can be introduced as an ideal current source not subject to resistive losses $\hat{I}$. In this case, the circuit equation is

$V_{\mathrm{b}}=R(I-\hat{I})+\mu_{0} \frac{r_{0} I}{2} \frac{\mathrm{d} l_{\mathrm{i}}}{\mathrm{d} t}+\mu_{0} r_{0} l_{\mathrm{i}} \frac{\mathrm{d} I}{\mathrm{~d} t}$

where the equivalent non inductive current $\hat{I}$ is defined as

$\hat{I}=\frac{\hat{V}}{R}=I \cdot \frac{\int \eta j_{\phi} \hat{j}_{\phi} \alpha \mathrm{d} V}{\int \eta j_{\phi}^{2} \mathrm{~d} V}$ 
For most Tokamaks, the total plasma current $I$ is kept constant by an external feedback mechanism that links the plasma current $I$ to the transformer voltage $V_{\mathrm{t}}$. If the total plasma current is kept constant, the ratio of the loop voltage before and after a non-inductive current is driven in the plasma (once stationary conditions are reached) is given by

$\frac{\Delta V}{\Delta V_{0}} \simeq \frac{\Delta R}{R_{0}}-\frac{\hat{I}}{I}$

The relative reduction of the loop voltage therefore gives an indication of the relative change in the plasma resistance and non-inductive current drive [2].

In stationary conditions $V=R(I-\hat{I})$, a reduction of the plasma resistance alone cannot lead to zero loop voltage, as this would imply zero plasma resistance. Only a non-inductive current can reduce the loop voltage to zero when all the current is driven non-inductively, $\hat{I}=I$. In a intermediate situation, the reduction of the loop voltage is due to both effects, noninductive current drive and plasma resistance reduction.

In an overdrive situation, the non-inductive current can exceed the set value for the total plasma current, in which case the transformer will counteract it. This situation is referred as transformer recharging. During recharging the loop voltage can be negative.

The loop voltage is most suitable for non-inductive current control when full current drive without transformer recharging is desired. In any other case the effects of both plasma heating and current drive can produce a reduction of the loop voltage, and they cannot be distinguished.

\subsection{Loop voltage state space model}

The relationship between the loop voltage output and the lower hybrid power input is best described by a system of first-order differential equations using an auxiliary vector $\boldsymbol{X}=\left(x_{1}, x_{2}\right.$, $\left.x_{3}\right)^{\mathrm{T}}$, where the variables $x_{1}, x_{2}, x_{3}$ are chosen to correspond with physical meaningful quanti- ties such as plasma resistance, internal inductance and ohmic current.

$$
\begin{aligned}
& x_{1}=\left\langle T_{\mathrm{e}}{ }^{-3 / 2}\right\rangle \\
& x_{2}=l_{\mathrm{i}} \\
& x_{3}=I-\hat{I}
\end{aligned}
$$

The first state is assumed to be proportional to the plasma resistance (Eq. 4), and is defined as

$\left\langle T_{\mathrm{e}}^{-3 / 2}\right\rangle=\frac{A}{2 \pi r_{0}} \frac{\int T_{\mathrm{e}}^{-3 / 2} j_{\phi}^{2} \mathrm{~d} V}{I^{2}}$

where $A$ is the area of the plasma cross-section.

This type of description is known as state space description, and the auxiliary variables $x_{\mathrm{i}}$ are known as internal states. This description is particularly useful for our purpose, as it allows physical mechanisms in the plasma to be incorporated in the model.

Using the state space description, the loop voltage measurement equation for constant plasma current $V=R(I-\hat{I})+I(\mathrm{~d} L / \mathrm{d} t)$ can be written as a non-linear output equation depending on the internal states $x_{1}, x_{2}, x_{3}$

$y=c_{1} x_{1} x_{3}+c_{2} \dot{x}_{2}$

The physical parameters are summarized in the model parameters through the expressions

$c_{1}=2 \pi \cdot 10^{-4} \frac{r_{0}}{A} \cdot \ln \Lambda \chi\left(Z_{\mathrm{eff}}\right)$,

$c_{2}=\mu_{0} \frac{r_{0}}{2} I$

where the dependence with the effective ionic charge is given by

$\chi\left(Z_{\text {eff }}\right)=Z_{\text {eff }}\left(0.29+\frac{0.457}{1.077+Z_{\text {eff }}}\right)$

and the Coulomb logarithm $\ln \Lambda$ is obtained from

$\Lambda=1.09 \cdot 10^{14} \frac{T_{\mathrm{e}}}{Z_{\mathrm{eff}} \sqrt{n_{\mathrm{e}}}}$

The numerical factors in $c_{1}$ are valid for an Spitzer resistivity model [10], with $T_{\mathrm{e}}$ in $\mathrm{eV}, \eta$ in ohms per metre, and $n_{\mathrm{e}}$ in electrons per cubic 
metre. A proportionality factor $c_{1}$ can still be assumed when neoclassical [11] and/or Fisch [12] corrections are introduced.

Physically,

$c_{1} x_{1} x_{3}=R(I-\hat{I}) \quad$ and $\quad c_{2} \dot{x}_{2}=\mu_{0} \frac{r_{0}}{2} I \frac{\mathrm{d}}{\mathrm{d} t}\left(l_{\mathrm{i}}\right)$,

therefore, the output equation is simply the relationship (Eq. 16) for constant plasma current.

Using the Akaike's information criterion [13], a first order lag is found to be the best approximation for the internal states $x_{\mathrm{i}}$ dynamics at JET [14]. Based on this, a first order model will be parametrized using system identification techniques. The system equations describing the dynamics of the plasma resistance $\left(\cong x_{1}\right)$, inductance $\left(x_{2}\right)$ and ohmic current $\left(x_{3}\right)$ can be written as

$\dot{x}_{\mathrm{i}}=-\frac{1}{\tau_{\mathrm{i}}}\left(x_{\mathrm{i}}-\tilde{x}_{\mathrm{i}}\right)+\frac{k_{\mathrm{i}}}{\tau_{\mathrm{i}}} u$,

where first order linear approximations for the internal state's $\left(x_{\mathrm{i}}\right)$ dynamics around a certain operating point $\tilde{x}_{\mathrm{i}}$ have been made. $k_{\mathrm{i}}$ and $\tau_{\mathrm{i}}$ are gains and time constants corresponding to $x_{\mathrm{i}}$

The internal states $x_{\mathrm{i}}$ must be coupled to some extent, as they all depend on the current profile (Eqs. 4, 17 and 8). As a first approximation, this coupling will be ignored.

The input vector to the system is the lower hybrid power delayed by $t_{\mathrm{d}}$,

$u(t)=P_{\mathrm{LH}}\left(t-t_{\mathrm{d}}\right)$

The gains $k_{\mathrm{i}}$ can be estimated from the change in the states $x_{\mathrm{i}}$ once stationary conditions are reached

$k_{\mathrm{i}}=\frac{\Delta x_{\mathrm{i}}}{P_{\mathrm{LH}}}=\frac{\left.x_{\mathrm{i}}\right|_{t \rightarrow \infty}-\left.x_{\mathrm{i}}\right|_{t=0}}{P_{\mathrm{LH}}}$

The gain $k_{1}$ at JET is always negative, as the lower hybrid power input always raises the electron temperature, and as a result, lowers $T_{\mathrm{e}}{ }^{-3 / 2}$. The time constant $\tau_{1}$ depends on combined heat transport and current diffusion mechanisms in the plasma, and can range from a tenth of a second to a few seconds.

The gain $k_{2}$ depends on the difference between the power deposition profile and the plasma cur- rent profile. When these profiles are different, the plasma current will diffuse in order to achieve a constant electric field across the plasma [15]. For instance, an initially peaked plasma current profile will be broadened by off-axis lower hybrid power deposition. Current profile broadening causes a reduction in the internal inductance $\left(x_{2}\right)$, and corresponds with $k_{2}$ being negative. The time constant $\tau_{2}$ is basically the skin time $\tau_{\mathrm{s}}$, and depends strongly on the plasma temperature. For a cylindrical plasma of minor radius $a$ and constant plasma resistivity $\eta$

$\tau_{\mathrm{s}}=\frac{\mu_{0} a^{2}}{\eta \lambda_{1}^{2}}$

where $\lambda_{1} \cong 3.831$ is the first zero of the $J_{1}$ Bessel function. For JET plasmas with typical electron temperatures between 1 and $10 \mathrm{KeV}$, the skin time varies between 0.5 and $5 \mathrm{~s}$. The skin time depends on the plasma resistivity, and must therefore be correlated with the plasma resistance $\left(\cong x_{1}\right)$. This has not been considered in the model, and an effective time constant $\tau_{2}$ is adopted to encompass these effects.

The amount of non-inductive current drive $I_{\mathrm{LH}}$ produced when lower hybrid power $P_{\mathrm{LH}}$ is injected into the plasma can be characterized by means of the current drive efficiency $\eta_{\mathrm{CD}}$, defined as

$\eta_{\mathrm{CD}}=\left\langle\eta_{\mathrm{e}}\right\rangle r_{0} \frac{I_{\mathrm{LH}}}{P_{\mathrm{LH}}}$

where $\left\langle\eta_{\mathrm{e}}\right\rangle$ is the volume-averaged electron density. Due to the total plasma current being kept constant by a feedback mechanism, the ohmic current gain $k_{3}$ is the same (in absolute value) as the non-inductive current drive gain, and can be expressed as a function of the non-inductive current drive efficiency $\eta_{\mathrm{CD}}$,

$k_{3}=-\frac{\eta_{\mathrm{CD}}}{r_{0} \eta_{\mathrm{e}}}$

The time constant $\tau_{3}$ describes how quickly the ohmic part of the plasma current $\left(x_{3}\right)$ decays when a non inductive current is created in the plasma. This should occur as a result of the plasma current feedback mechanism. For constant plasma current, inductance and resistance, the following relationship must hold 
$I-\hat{I}=\frac{-\frac{M_{\mathrm{T}}}{R} s}{\frac{L_{\mathrm{T}}}{R_{\mathrm{T}}} s+1} V_{\mathrm{T}}$

Since $\mathrm{L}_{\mathrm{T}} \simeq 180 \times 10^{-3} \mathrm{H}, R_{\mathrm{T}} \simeq 30 \times 10^{-6} \Omega$, the time constant of the transformer current in the primary winding is extremely slow, $L_{\mathrm{T}} / R_{\mathrm{T}} \simeq$ $6000 \mathrm{~s}$, and Eq. (31) can be simplified to

$I-\hat{I} \simeq-\frac{M_{\mathrm{T}}}{R} \frac{R_{\mathrm{T}}}{L_{\mathrm{T}}} V_{\mathrm{T}}$

Therefore, for constant plasma current, inductance and resistance, the time constant involved in the ohmic current decay is the same as the flywheel generator output voltage $V_{\mathrm{T}}$ response time. For the flywheel generator at JET, this is $\sim 0.15 \mathrm{~s}$ [16].

There are many discharges in which stationary conditions are not attained, and it is therefore difficult to obtain the model parameters using Eq. (27). In these cases, the model parameters $k_{\mathrm{i}}, \tau_{\mathrm{i}}$ can be estimated from the measured time traces $\tilde{x}_{1}, \tilde{x}_{2}, \tilde{y}$ by minimizing the loss function

$\varepsilon\left(k_{\mathrm{i}}, \tau_{\mathrm{i}}\right)=\varepsilon\left(k_{\mathrm{i}}, \tau_{\mathrm{i}}\right)=|y-\tilde{y}|+\sum_{i=1,2}\left|x_{\mathrm{i}}-\tilde{x}_{\mathrm{i}}\right|$,

with respect to $k_{\mathrm{i}}, \tau_{\mathrm{i}}$. The model output $y$ and internal states $x_{\mathrm{i}}$ are calculated from Eq. (21) and Eq. (25). This parameter estimation technique is known in the literature as the output error method (OE) [5].

Figs. 4 and 5 demonstrate a fitting example for the states $x_{\mathrm{i}}$ and the resulting loop voltage compared with the actual data. The third state (ohmic current) is not shown due to it being directly related to the lower hybrid power input, with a fixed time constant $\tau_{3}=0.15 \mathrm{~s}$. The parameters obtained were

$k_{1}=-3.2 \times 10^{-12} \mathrm{eV}^{-3 / 2} \mathrm{~W}^{-1}$,

$k_{2}=-0.024 \times 10^{-6} \mathrm{~W}^{-1}$,

$\eta_{\mathrm{CD}}=1.18 \times 10^{19} \mathrm{~m}^{-2} \mathrm{~A} \mathrm{~W}^{-1}$,

$\tau_{1}=0.04 \mathrm{~s}$,

$\tau_{2}=0.05 \mathrm{~s}$.
The physical parameters of the discharge were

$r_{0}=2.96 \mathrm{~m}$,

$I=2.5 \cdot 10^{6} \mathrm{~A}$,

$Z_{\text {eff }}=2.5$,

$n_{\mathrm{e}}=2 \cdot 10^{19} \mathrm{~m}^{-3}$.

One of the most important parameters in this model is the current drive efficiency. The experimentally determined current drive efficiency at JET increases with the electron temperature [17], therefore, in principle, the gain $k_{3}$ must also be correlated with $x_{1}$. This has not been taken into account in the state space model, and the effective steady state gain $k_{3}$ is assumed to combine these effects.

\subsubsection{Noise and perturbations}

The output equation (Eq. 21) was obtained from the loop voltage measurement equation assuming constant plasma current. However, according to Eq. (13), small oscillations of the plasma current around its equilibrium value introduce an additive perturbation $w$ in the loop voltage given by

$w=\mu_{0} r_{0}\left(l_{\mathrm{i}}+l_{\mathrm{e}}\right) \frac{\mathrm{d} I}{\mathrm{~d} t}$

This perturbation can be seen in Fig. 5 as a small oscillation of the loop voltage signal that is not reproduced in the simulation.

The loop voltage also has a component due to the plasma movement. If $\psi$ is the flux relative to the flux surface corresponding with the measuring loop, and $u$ is the velocity of this flux surface relative to the location of the measuring loop, then the convective time derivative gives the measured loop voltage as:

$V_{1}=-2 \pi \frac{\mathrm{d} \psi}{\mathrm{d} t}=-2 \pi\left(\frac{\partial \psi}{\partial t}+(\mathbf{u} \cdot \nabla) \psi\right)$

To distribute the power load in the divertor tiles, the $x$-point is sometimes made to oscillate. This is achieved by modulating the current in the divertor coils with a frequency of $4 \mathrm{~Hz}$. This produces a movement of the flux surfaces relative to the measuring loop. As a consequence of this movement, a low frequency noise appears in the loop voltage and indeed in almost all magnetic diag- 


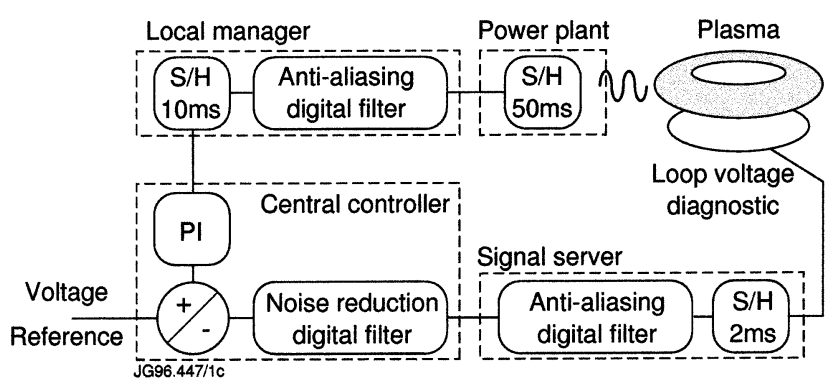

Fig. 6. Loop voltage feedback loop.

nostics. The solution to this consists of strong filtering of the loop voltage signal with a $1 \mathrm{~Hz}$ cutoff low pass filter, whose equivalent transfer function is

$F(s)=\frac{38}{s^{2}+9 s+38}$,

where $s$ is the Laplace operator. The divertor has been replaced recently by the Mark II divertor [18], with better power handling that makes the divertor sweep unnecessary.

\subsection{Loop voltage transfer function}

The former non-linear state space model can be approximated by a linear transfer function with the same frequency response at low frequencies, in the form

$G(s)=\frac{V(s)}{P_{\mathrm{LH}}(s)} \simeq F(s)\left(\frac{k_{\mathrm{res}}+s k_{\mathrm{der}}}{\tau_{\mathrm{s}}}\right) \mathrm{e}^{-t_{\mathrm{d}} s}$

where it has been considered that $\tau_{1} \simeq \tau_{2} \simeq \tau_{\mathrm{s}}$, $\tau_{3} \simeq 0$. The resistive loop voltage gain $k_{\text {res }}$ is

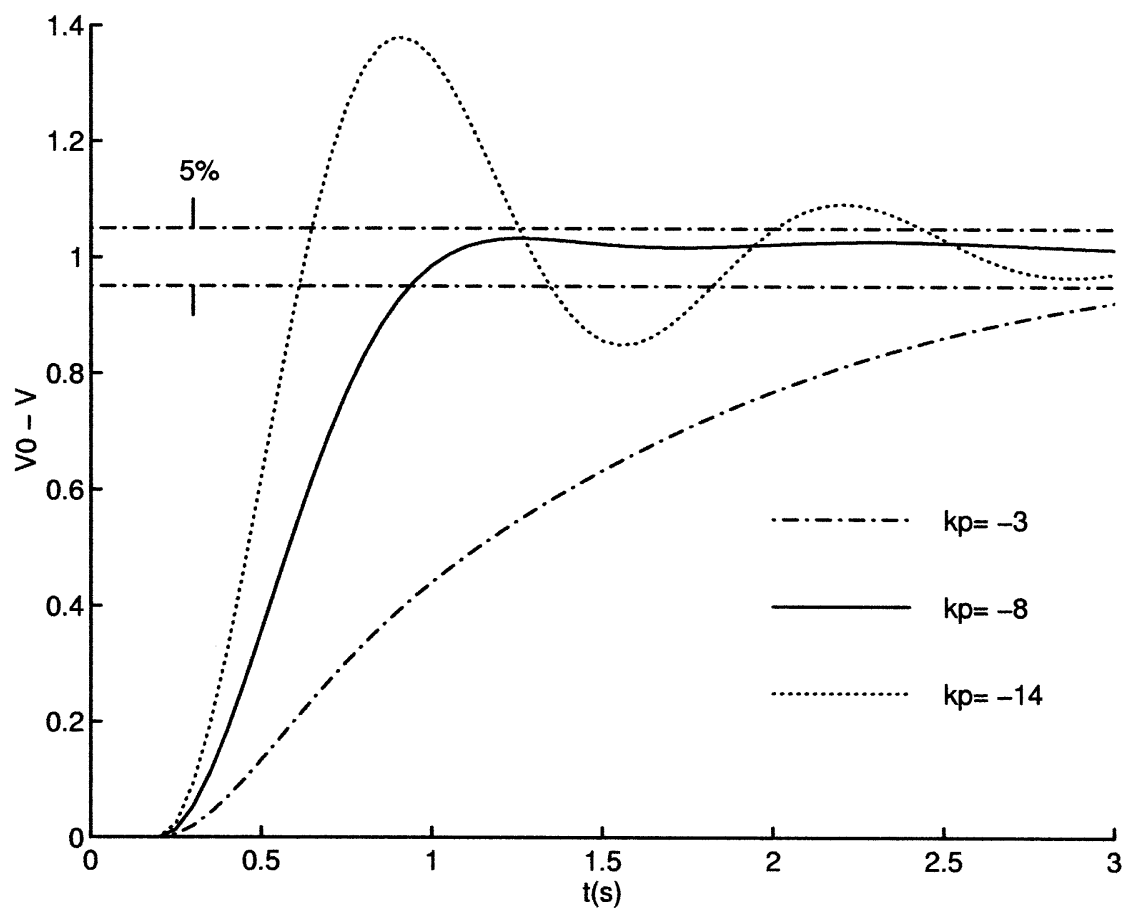

Fig. 7. Simulation of the loop voltage control system step response for three proportional gain values. 

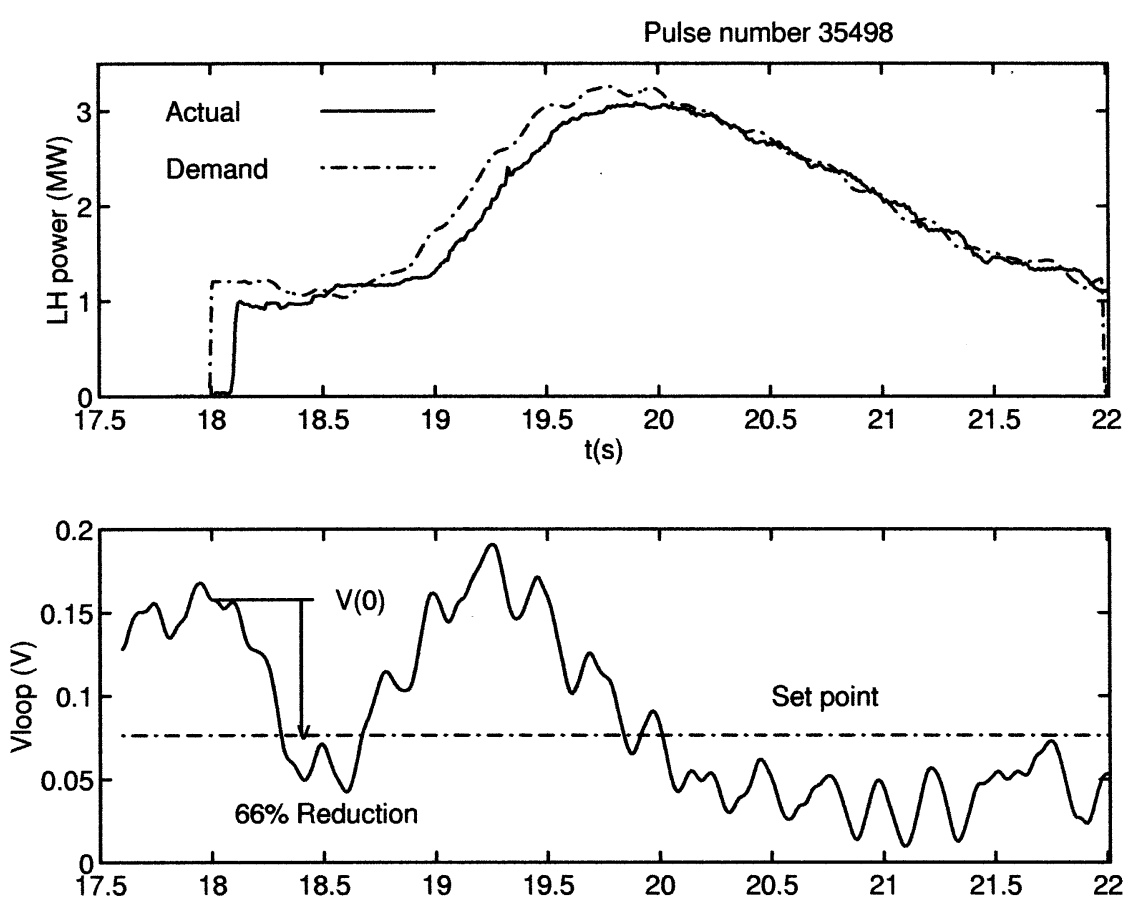

Fig. 8. Loop voltage control with $k_{\mathrm{p}}=-3$.

$$
\begin{aligned}
k_{\text {res }} & =c_{1}\left(\left.\hat{x}_{3}\right|_{t=0} k_{1}+\left.\tilde{x}_{1}\right|_{t=0} k_{3}\right) \\
& =\left.V^{\mathrm{res}}\right|_{t=0}\left(\frac{\Delta\left\langle T_{\mathrm{e}}^{-3 / 2}\right\rangle}{\left\langle T_{\mathrm{e}}^{-3 / 2}\right\rangle_{t=0} \Delta P_{\mathrm{LH}}}-\frac{\eta_{\mathrm{CD}}}{r_{0} \eta_{\mathrm{e}} I}\right)
\end{aligned}
$$

where the resistive loop voltage initial value $\left.V^{\text {res }}\right|_{t=0}$ is calculated just before lower hybrid power is applied,

$\left.V^{\mathrm{res}}\right|_{t=0}=\left.V\right|_{t=0}-\left.\frac{\partial}{\partial t}(\mathrm{LI})\right|_{t=0}$,

and the gain $k_{\text {der }}$ accounts for the derivative contribution to the loop voltage,

$$
\begin{aligned}
k_{\mathrm{der}} & =c_{2} k_{2}+\left.c_{1} \tau_{\mathrm{s}} \tilde{x}_{1}\right|_{t=0} k_{3} \\
& =\mu_{0} \frac{r_{0}}{2} I \frac{\Delta l_{i}}{\Delta P_{\mathrm{LH}}}-\left.V^{\mathrm{res}}\right|_{t=0} \frac{\eta_{\mathrm{CD}}}{r_{0} \eta_{\mathrm{e}} I} \tau_{\mathrm{s}}
\end{aligned}
$$

The term $\Delta\left\langle T_{\mathrm{e}}{ }^{-3 / 2}\right\rangle /\left\langle T_{\mathrm{e}}{ }^{-3 / 2}\right\rangle_{t=0} \Delta P_{\mathrm{LH}}$ represents the relative reduction in the plasma resistance per unit of lower hybrid power, and $\Delta l_{\mathrm{i}}$ is the internal inductance reduction when $\Delta P_{\mathrm{LH}}$ lower hybrid power is applied.

\subsection{Loop voltage control}

The objective of loop voltage control is to regulate the non-inductive current drive in the plasma. The experimental conditions for the first control experiments are chosen to generate enough non-inductive current in the plasma to obtain a substantial loop voltage reduction. The maximum lower hybrid power to be used in the experiments is limited to $5 \mathrm{MW}$. Considering an average electron temperature $\left\langle T_{\mathrm{e}}\right\rangle=1.5 \times$ $10^{3} \mathrm{eV}$, the current drive efficiency is $\eta_{\mathrm{CD}}=1.6 \times$ $10^{19} \mathrm{~A} \mathrm{~m}^{-2} \mathrm{~W}^{-1}$ [17]. In order to obtain $50 \%$ of the plasma current driven non-inductively in a discharge with $I=2.5 \cdot 10^{6} \mathrm{~A}$ and $P_{\max }=5 \mathrm{MW}$, a density lower than $\eta_{\mathrm{e}}<2 \cdot 10^{19} \mathrm{~m}^{-3}$ is necessary (Eq. 29). The averaged plasma density volume is therefore chosen as

$\left\langle\eta_{\mathrm{e}}\right\rangle=2 \cdot 10^{19} \mathrm{~m}^{-3}$

When a residual (ohmic) electric field is present in the plasma, the fast electrons can be accelerated towards higher energies by the slide-away effect. 
In contrast with the runaway situation, the electrons are not accelerated indefinitely, however, an upper energy limit exists determined by the ParailPogutse instability [19]. This effect can alter the dynamics of the fast electrons and potentially perturb the experimental conditions. To avoid the slide-away effect, the residual electric field responsible for the acceleration of the fast electrons must be reduced. This can be achieved by choosing a significant reduction of the loop voltage, ideally $V=0$, which guarantees full non inductive current drive and avoids transformer recharging.

The loop voltage controller design will be based on the following experimental conditions

$I=2.5 \cdot 10^{6} \mathrm{~A}$

$n_{\mathrm{e}}=2 \cdot 10^{19} \mathrm{~m}^{-3}$

$\left\langle T_{\mathrm{e}}\right\rangle \simeq 1.5 \cdot 10^{3} \mathrm{eV}$

\subsubsection{Controller design}

In this section, the design for the loop voltage $V_{1}$ controller is presented. As seen in the previous section, the model for $V_{1}$ does not have a pure integrator. Therefore, a proportional integral (PI) controller is the minimum requirement in order to achieve control without steady state error. The PI controller designed in this section will serve as a starting point for the first control experiments.

The transfer function between the loop voltage and the lower hybrid power demand is obtained from Eq. (39)

$G(s)=\left(1-R_{\mathrm{c}}\right) F(s)\left(\frac{k_{\mathrm{res}}+s k_{\mathrm{der}}}{\tau_{\mathrm{s}} s+1}\right) \mathrm{e}^{-t_{\mathrm{d}} s}$

where $R_{\mathrm{c}}$ is the reflection coefficient, e.g. the fraction of lower hybrid power reflected back from the plasma. $R_{\mathrm{c}}$ is an exponential function of the distance between the LH antenna and the plasma boundary, and is subject to uncertainties and perturbations. For this reason, $R_{\mathrm{c}}$ is usually feedback controlled at 3\% $\left(R_{\mathrm{c}}=0.03\right)$ by movement of the LH launcher around an equilibrium distance from the plasma [20].

The controller is to be used in a semi-stationary situation where the plasma current is kept constant and the plasma current profile is not changing. At the initial state before the feedback loop is closed, the derivative contribution is negligible and the initial value of the loop voltage is only resistive. Taking $\mathrm{V}_{0}^{\text {res }}=\mathrm{V}_{0}=0.2 \mathrm{~V}$, and evaluating the parameters $k_{\text {res }}, k_{\text {der }}$ (Eqs. 40 and 42 ) with the experimental conditions (Eq. 43), the transfer function parameters (Eq. 39) are obtained as

$$
\begin{aligned}
& k_{\text {res }}=-0.05 \times 10^{-6} \mathrm{~V} \mathrm{~W}^{-1}, \\
& k_{\text {der }}=-0.04 \times 10^{-6} \mathrm{~V} \mathrm{~W}^{-1} \\
& \tau_{\mathrm{s}}=1 \mathrm{~s}, \\
& t_{\mathrm{d}}=0.12 \mathrm{~s}
\end{aligned}
$$

and the transfer function used for the design is

$$
G(s) \simeq-10^{-6} F(s)\left(\frac{0.04 s+0.05}{s+1}\right) \mathrm{e}^{-0.12 \mathrm{~s}}
$$

The control system structure is shown in Fig. 6 . The loop voltage is obtained from the loop placed on the bottom of the machine and outside the vessel.

A PI (proportional integral) controller is used for the design. The equivalent transfer function of the PI controller in the continuous time domain is

$$
C(s)=k_{\mathrm{p}}\left(1+\frac{1}{s T_{\mathrm{i}}}\right)
$$

The proportional gain $k_{\mathrm{p}}$ and integral term $T_{\mathrm{i}}$ are selected to obtain the maximum phase and gain margins for the closed loop system without making it too slow [21]. The integral term set to $T_{\mathrm{i}}=0.2 \mathrm{~s}$. Using the discrete equivalent system with $T=50 \mathrm{~ms}$, a critical gain of $k_{\mathrm{c}}=-24$ is calculated. If $k_{\mathrm{p}}<k_{\mathrm{c}}$, the closed system will be unstable. Fig. 7 shows the step response of the closed loop system for several values of the proportional gain $k_{\mathrm{p}}>k_{\mathrm{c}}$. The response corresponding with $k_{\mathrm{p}}=-8$ lies within $5 \%$ of the reference value in $\sim 1.5 \mathrm{~s}$. The phase $P_{\mathrm{m}}$ and amplitude $G_{\mathrm{m}}$ margins of the closed loop system corresponding with $k_{\mathrm{p}}=-8$ are

$G_{\mathrm{m}}=9.5 \mathrm{~dB}$

$P_{\mathrm{m}}=62^{\circ}$

\subsubsection{Experimental results}

In order to adjust the gain in the feedback loop, three control experiments were performed. To 

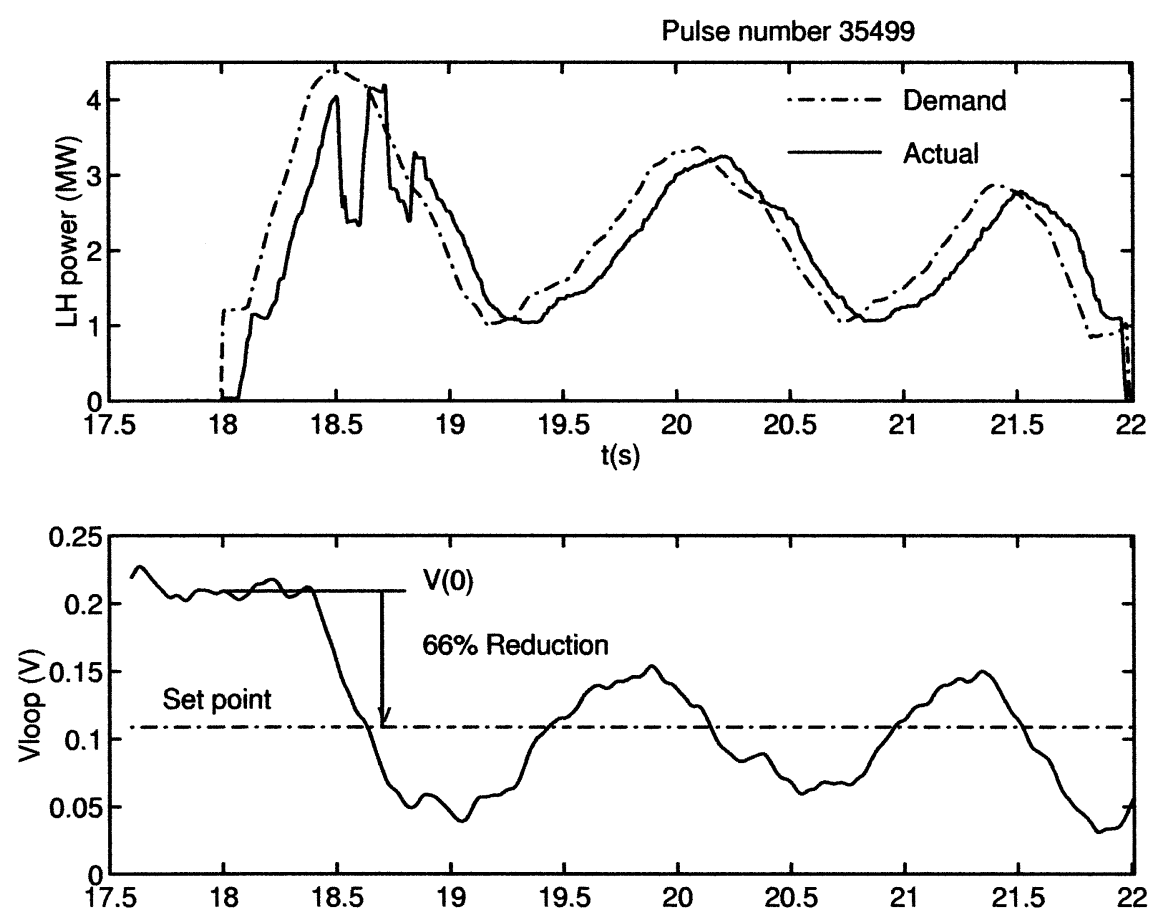

Fig. 9. Loop voltage control with a gain very close to the critical one, $k_{\mathrm{p}}=-14$.

obtain the same conditions for the three experiments, the reference for the loop voltage was set as a percentage reduction of the loop voltage just before feedback $\left(\left.V\right|_{t=0}=0.2 \mathrm{~V}\right)$. This percentage was fixed at $66 \%$.

The critical gain, $k_{\mathrm{c}}=-24$, calculated in the previous section gives a starting point for the first control experiments. The first experiment was performed with a loop gain, $k_{\mathrm{p}}=-3$, well below the critical gain. Accordingly, the closed loop shows a very slow response (Fig. 8).

The second experiment (Fig. 9) was performed setting the loop gain at the expected critical value, $k_{\mathrm{p}}=-14$. The system shows oscillatory behaviour with an almost constant amplitude corresponding to the loop gain being very close to the critical one.

For the third experiment (Fig. 10), an intermediate value of the loop gain was chosen, $k_{\mathrm{p}}=-7$. The loop voltage approaches the reference $(66 \%$ reduction) with an underdamped behaviour $(33 \%$ overshoot).

\section{Off-axis current control}

Recently, off-axis current control has become an important issue for shear optimization experiments. To improve the performance of shear-reversed discharges, a very broad hollow plasma current profile is needed [22]. This is usually achieved by ramping up the plasma current at a faster rate than the skin time, which creates an off-axis current distribution. By subsequent heating of the plasma core, the off-axis current is prevented from diffusing inwards, due to the lower skin time associated with a hotter plasma [23]. This may result in a low or even negative shear over a central region of the plasma, which can reduce the plasma turbulence, and therefore increase the confinement [24].

However, there are many uncertainties and perturbations that may lead to an off-axis current over / under drive, and failure to achieve the target current profile configurations. As the off-axis current over/under-drive is not easily predicted, pre- 

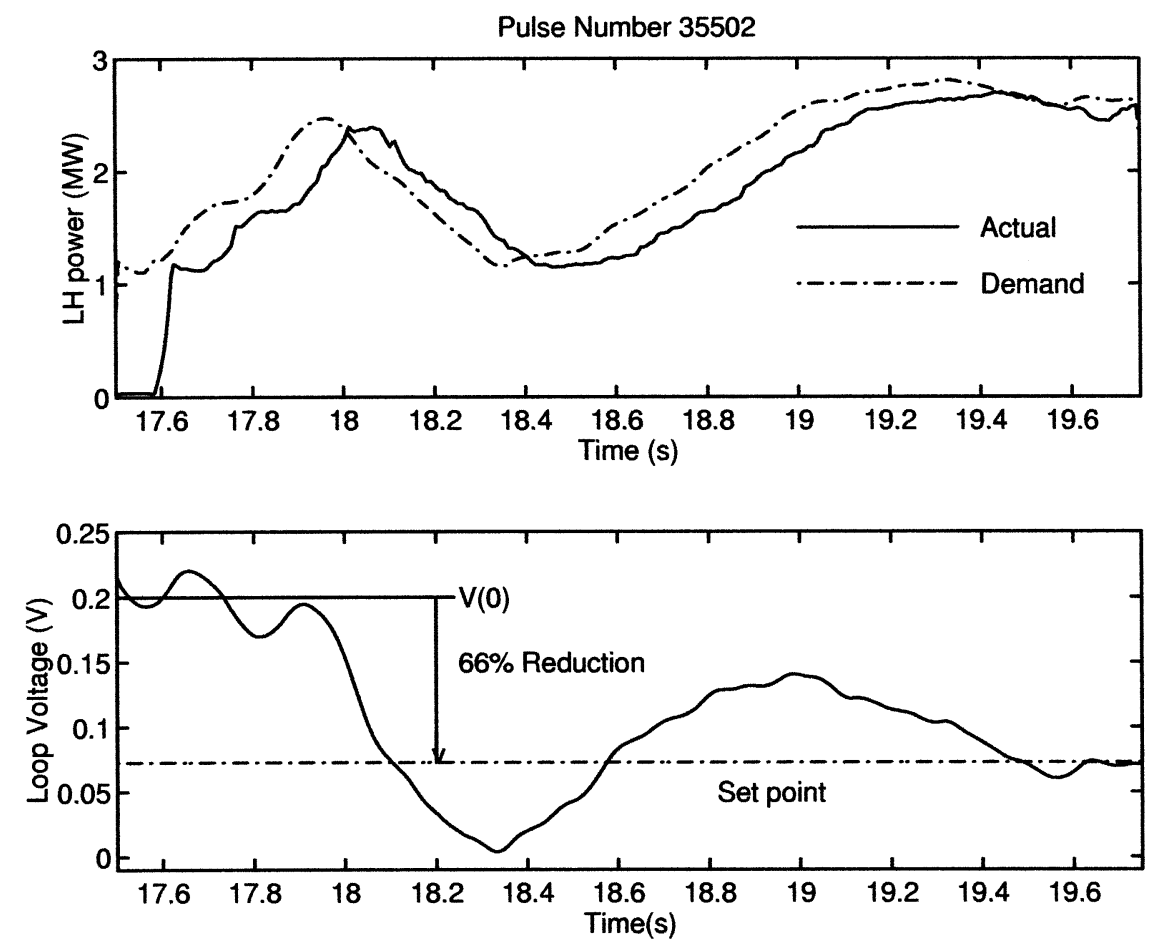

Fig. 10. Loop voltage control with $k_{\mathrm{p}}=-7$.

programming of the ramp rate to control the off-axis current is cumbersome, and numerous discharges are required for commissioning.

An improved approach is to control the off-axis plasma current being generated directly, so that instead of imposing waveforms for the current ramps, reference waveforms are imposed in the desired off-axis current.

The second moment of the plasma current distribution contains information about the off-axis current profile distribution, and feedback control of the second current moment can be used for off-axis current regulation.

Lower hybrid waves can also be used to help broaden the profile during the current raise phase. In this section, the design stages and experimental results of the second current moment $Y_{2}$ control using lower hybrid waves are presented.

\subsection{Second current moment definition}

Traditionally, low order current moments of the plasma current distribution have been used to obtain global plasma parameters such as the total plasma current $I$ (zero order moment) or the coordinates $\left(r_{0}, z_{0}\right)$ of the plasma current centroid (first current moment).

The second current moment of a current distribution is defined as

$y_{2}=\frac{\int_{\Omega} j \phi f_{2} \mathrm{~d} A}{I}$

In our case, the integration region $\Omega$ is the plasma section bounded by the LCFS. $I$ is the total plasma current, and $f_{2}$ is a weighting on the current density profile. The weighting $f_{2}$ is chosen to satisfy the homogeneous Shafranov equation

$\Delta * f_{2}=0$

and is given by

$f_{2}=\left(\frac{r-r_{0}}{2 r_{0}}+1\right)^{2}\left(r-r_{0}\right)^{2}-\left(\frac{r-r_{0}}{r_{0}}+1\right)^{2}\left(z-z_{0}\right)^{2}$ 
The weighting function $f_{2}$ increases in value with the distance from the plasma current centroid, resulting in a higher weighting for the off-axis region of the current distribution. If the integration region is bounded by the plasma boundary, the integral (Eq. 49) can be expressed as a line integral of the poloidal magnetic field $B_{\theta}$ along the plasma boundary $\Gamma$

$y_{2}=\frac{\oint_{\Gamma} f_{2} B_{\theta} \mathrm{d} l}{\mu_{0} l}$

Due to the asymmetry in the $z$ and $r$ components in the weighting $f_{2}$, (Fig. 11), the integral in Eq. (49) is sensitive to the plasma elongation. This was first realized by Zakharov and Shafranov [25], who proposed the second current moment for plasma elongation determination. However, since the weighting $f_{2}$ increases with the distance to the plasma current centroid, the plasma current density profile $j_{\phi}$ has a higher weighting in the off-axis region, and the integral (Eq. 49) also contains information regarding the off-axis cur-

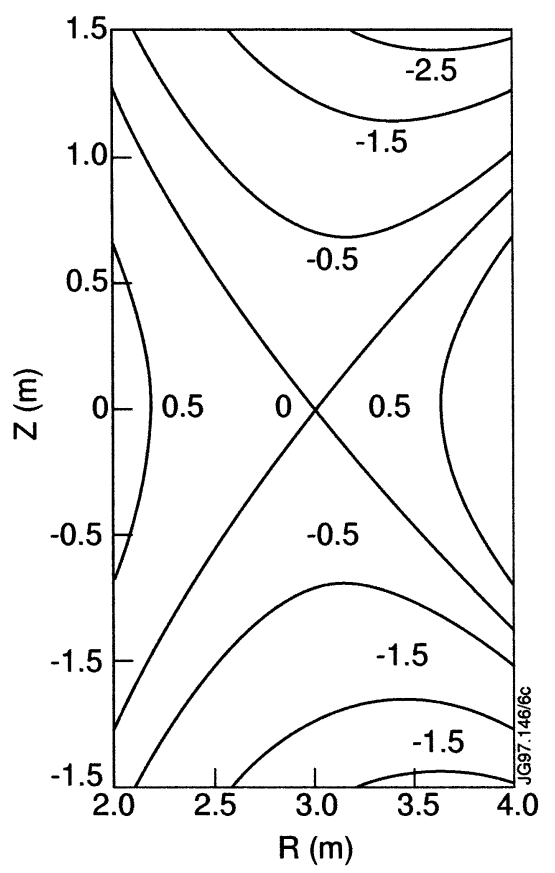

Fig. 11. Contour plot for the weighting function $f_{2}$ with $r_{0}=3$ $\mathrm{m}, z_{0}=0$. rent profile distribution. The integral (Eq. 49) is sensitive to both plasma elongation and off axis current, and the relative influence of each contribution is left undetermined. A straightforward solution to this problem is to normalize Eq. (49). The normalized second current moment is then defined as

$Y_{2}=\frac{\int_{\Omega} j_{\phi} f_{2} \mathrm{~d} A}{I} \frac{\int_{\Omega} \mathrm{d} A}{\int_{\Omega} f_{2} \mathrm{~d} A}$

The normalization factor

$Y_{2}=\frac{\int_{\Omega} j_{\phi} f_{2} \mathrm{~d} A}{I} \frac{\int_{\Omega} \mathrm{d} A}{\int_{\Omega} f_{2} \mathrm{~d} A}$.

is introduced to obtain a dimensionless quantity that is independent of the plasma shape. This type of calculation is only possible in equilibrium reconstruction codes, as direct magnetic field measurements are not possible at the plasma boundary. In the following text, the second current moment refers to the normalized second current moment $Y_{2}$.

At JET, the second current moment is available in real time from a fast equilibrium reconstruction system [26]. This allows use of the second current moment for real time off-axis current control. Fig. 12 shows the typical evolution of the second current moment and internal inductance. When the off-axis current increases the internal inductance decreases and the second current moment increases. During the flat top of the discharge, second current moment and internal inductance contain similar information about the off-axis current profile.

However, the real time determination of the internal inductance at JET is very sensitive to errors on the plasma boundary reconstruction during the initial current rise phase of the discharge. Plasma pressure anisotropy can also lead to errors on the diamagnetic beta, $\beta$ calculation during the high power heating phase [27]. These errors in $\beta$ are additive to the $l_{\mathrm{i}}$ calculation [28]

In contrast, the second current moment calculation is very reliable both in the plasma current rise 


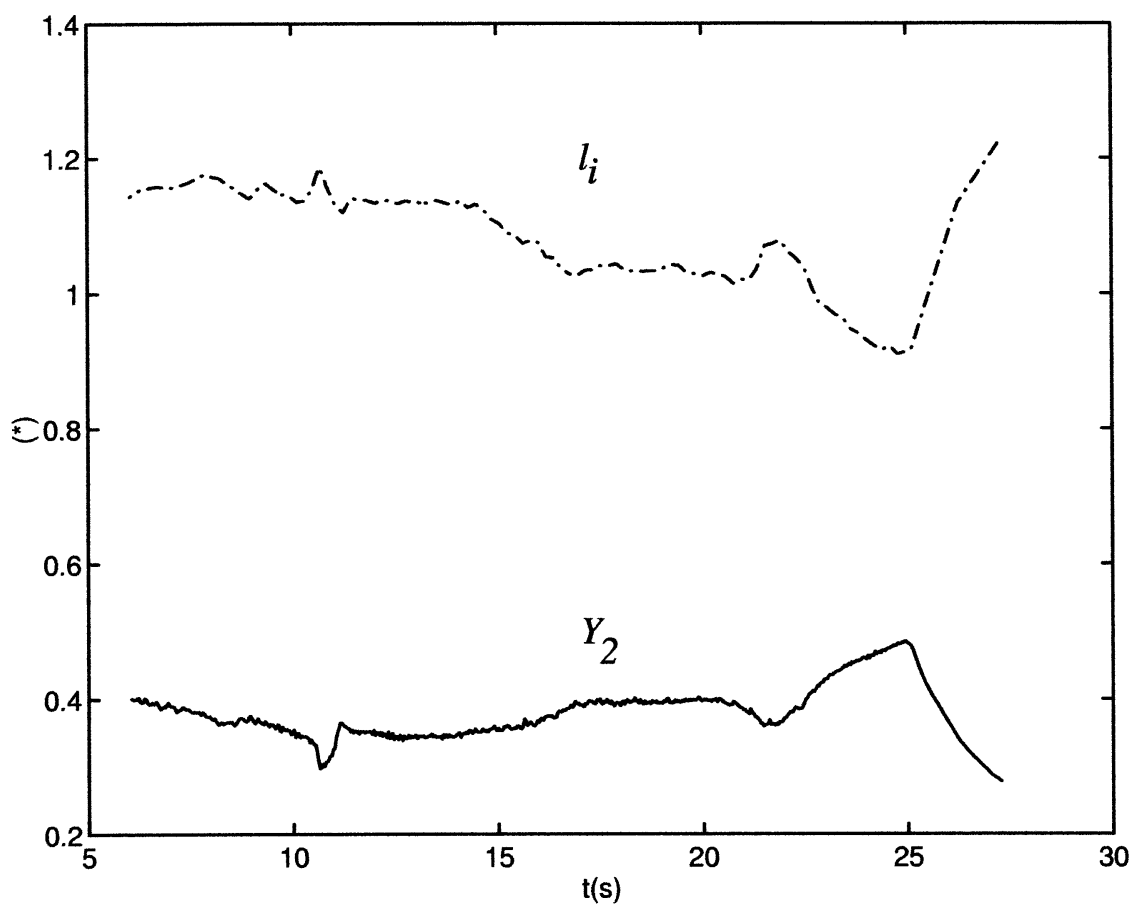

Fig. 12. Typical second current moment and internal inductance evolution during the flat top of the discharge (pulse 36918).

phase and plasma heating phase. For this reason the second current moment provides a more robust real time solution to off-axis current determination, being suitable for both current rise and plasma heating phases of the discharge.

\subsection{Second current moment model}

In order to design a control system for $Y_{2}$, it is necessary to obtain a model relating the power input to the control variable $Y_{2}$. To be able to use existing feedback control design methods, a model must be obtained in which the second current moment $Y_{2}$ is related to the power input through low order linear differential equations. A model described in this form must be able to reproduce the experimental time traces of $Y_{2}$ when the actual power input used in the experiment is used in the simulations. A model that fulfills all of these requirements will be derived in this section.

The simplest approximation for the dynamic relationship between lower hybrid power input and second current moment $Y_{2}$ is a first order differential equation in the form

$$
\tau_{\mathrm{s}} \dot{y}_{2}(t)+y_{2}(t)=k_{\mathrm{LH}} \cdot P_{\mathrm{LH}}(t)
$$

The gain $k_{\mathrm{LH}}$ depends on the difference between the power deposition profile and the plasma current profile, and can be estimated from the difference between the $Y_{2}$ value just before applying the power and the $Y_{2}$ value once stationary conditions are reached:

$k_{\mathrm{LH}}=\frac{\left.Y_{2}\right|_{t \rightarrow \infty}-\left.Y_{2}\right|_{t=0}}{P_{\mathrm{LH}}}$

The time constant is simply the skin time of the discharge $\tau_{\mathrm{s}}$.

The differential Eq. (53) can also be written as a first order transfer function between $P_{\mathrm{LH}}$ and $Y_{2}$

$Y_{2}(s)=\frac{k_{\mathrm{LH}}}{\tau_{s} s+1} P_{\mathrm{LH}}(s)$

where $s$ is the Laplace operator.

The model parameters $k_{\mathrm{LH}}, \tau_{\mathrm{s}}$ can be estimated from the measured time traces $\tilde{Y}_{2}$ and the model output $Y_{2}$ by minimizing the loss function

$\varepsilon\left(k_{\mathrm{LH}}, \tau_{\mathrm{s}}\right)=\left|Y_{2}-\tilde{Y}_{2}\right|$ 

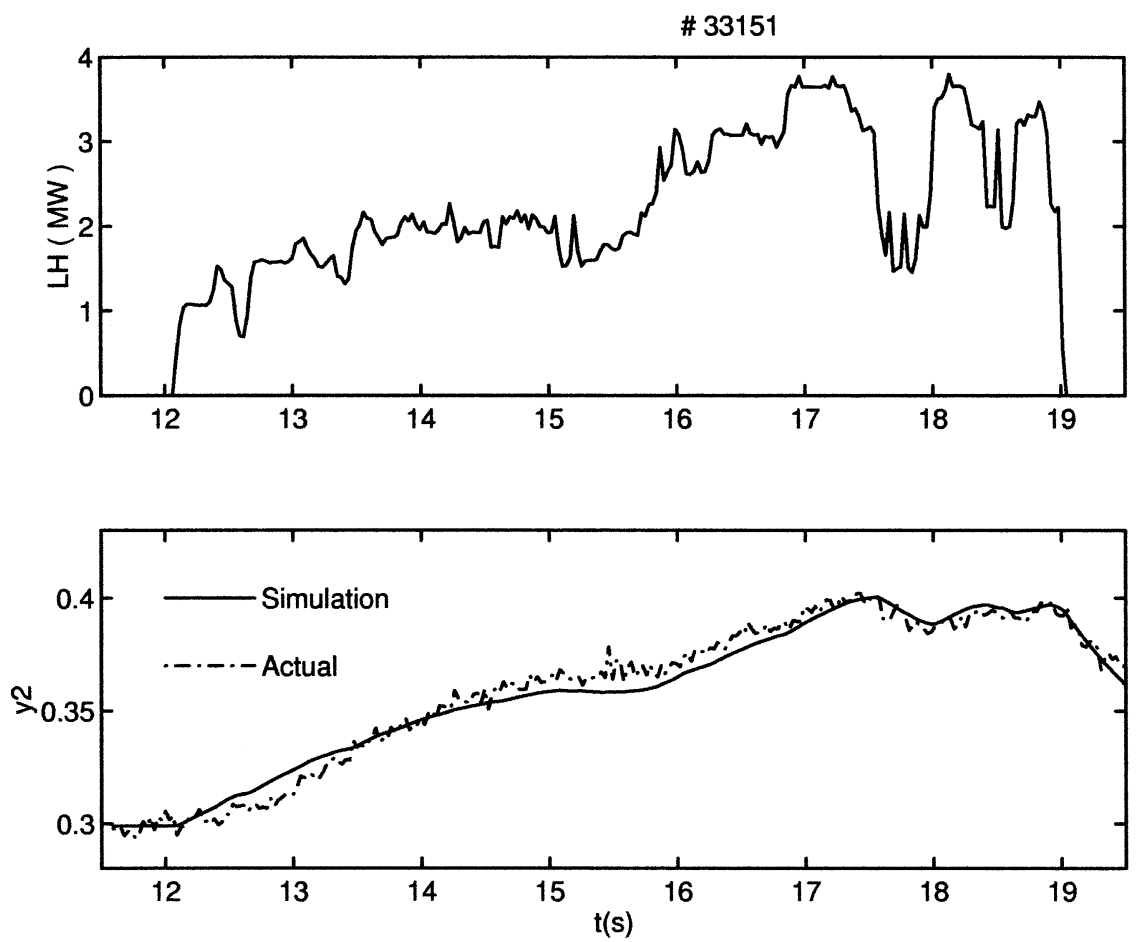

Fig. 13. Second current moment simulation with a first order differential equation. Time constant is $1.15 \mathrm{~s}$.

with respect to $k_{\mathrm{LH}}, \tau_{\mathrm{s}}$. The model output $Y_{2}$ is calculated from

$\dot{Y}_{2}=-\frac{1}{\tau_{\mathrm{s}}}\left(Y_{2}-\left.\tilde{Y}_{2}\right|_{t=0}\right)+\frac{k_{\mathrm{LH}}}{\tau_{\mathrm{s}}} P_{\mathrm{LH}}$

where the operation point $\left.\tilde{Y}_{2}\right|_{t=0}$ is chosen as the initial value of the second current moment just before lower hybrid power is applied. Fig. 13 shows a fitting example for shot 33151. The parameters obtained were $k_{\mathrm{LH}}=0.034 \mathrm{MW}^{-1}$ and $\tau_{\mathrm{s}}=1.15 \mathrm{~s}$. As seen from this example, the second current moment evolution due to the lower hybrid power is easily modelled by a first

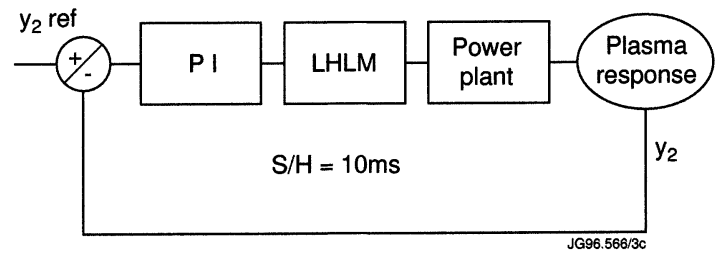

Fig. 14. Overview of the second current moment control system using lower hybrid waves. order differential equation with just two parameters, a gain $k_{\mathrm{LH}}$ and a time constant $\tau_{\mathrm{s}}$.

The model parameters have been found for two sets of experimental conditions of interest. These include:

(1) The initial plasma current raise phase just after the $x$-point formation of shear optimization experiments.

(2) The steady state phase of 3 MA discharges with volume averaged density $\left\langle\eta_{\mathrm{e}}\right\rangle \cong\left(2 \times 10^{19}\right)$ and temperature $\left(T_{\mathrm{e}}\right) \cong(1-2.5) \mathrm{KeV}$.

The parameters $k_{\mathrm{LH}}, \tau_{\mathrm{s}}$ obtained for these two scenarios are summarised in the table below:

\begin{tabular}{|c|c|c|c|c|}
\hline$I$ (MA) & $\left\langle n_{\mathrm{e}}\right\rangle$ & $\begin{array}{l}\left\langle T_{\mathrm{e}}\right\rangle \\
(\mathrm{KeV})\end{array}$ & $\begin{array}{l}k_{L H} \\
\left(\mathrm{~W}^{-1}\right)\end{array}$ & $\tau_{s}(\mathrm{~s})$ \\
\hline 3 & $2 \times 10^{19}$ & $1-2.5$ & $\begin{array}{l}0.035 \\
\times 10^{-6}\end{array}$ & $0.5-3$ \\
\hline $0.5 \rightarrow 2$ & $1 \times 10^{19}$ & 1 & $\begin{array}{l}0.060 \\
\times 10^{-6}\end{array}$ & 0.5 \\
\hline
\end{tabular}



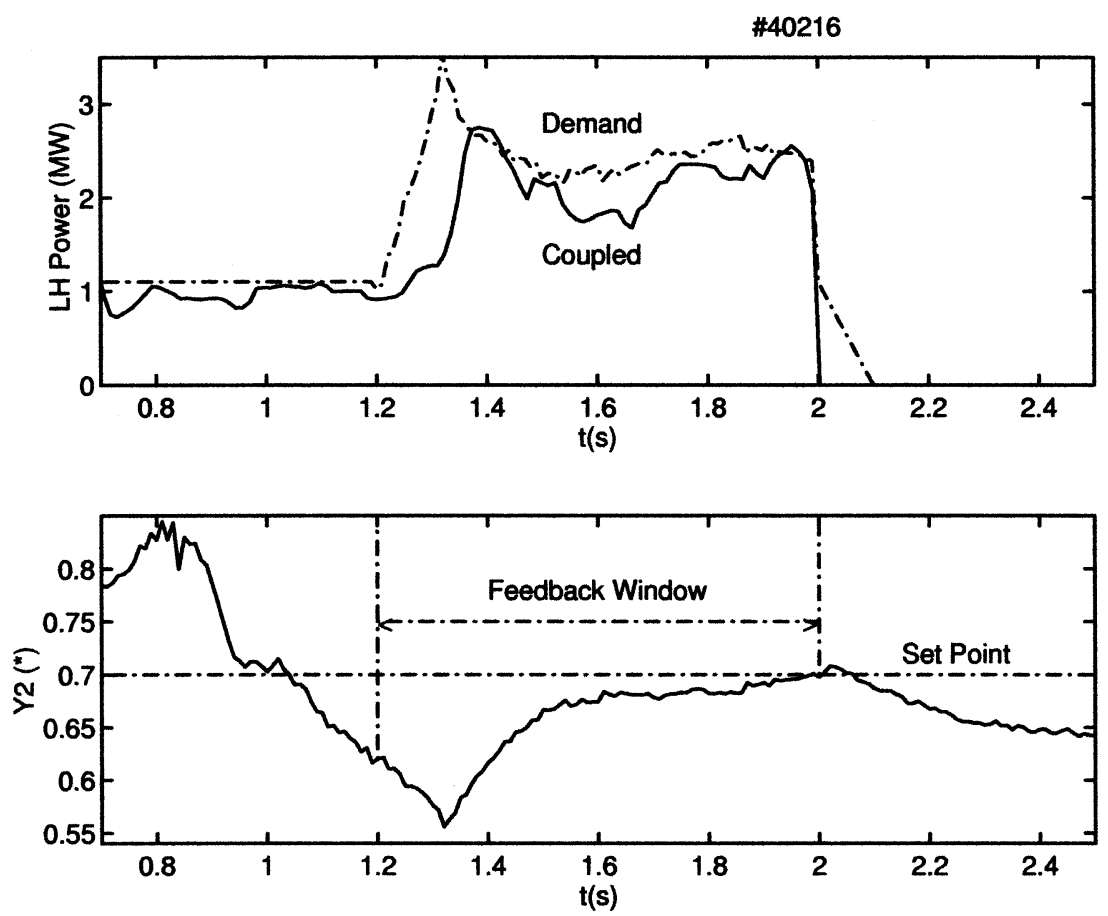

Fig. 15. Second current moment control with lower hybrid waves. Top: Power demanded by the PI controller (dot-dashed) and actual lower hybrid power (solid line). Bottom: Second current moment (solid line) and set point for feedback (dot-dashed).

The gain $k_{\mathrm{LH}}$ varies by $15 \%$ across all the pulses studied.

In the Section 4.3, a second current moment control system using lower hybrid will be designed based on the model parameters $k_{\mathrm{LH}}=0.06 \times$ $10^{-6} \mathrm{~W}^{-1}, \tau_{\mathrm{s}}=0.5$. This corresponds with the plasma current raise phase during shear optimization experiments at JET.

\subsection{Controller design}

In this section, the components of a second current moment control system will be designed for the plasma current rise phase of a shear reversal scenario. The control system is shown in Fig. 14. The LH plant has been upgraded recently and it is now interrupt-controlled with a fixed sampling time of $10 \mathrm{~ms}$.

The transfer function between $Y_{2}$ and the lower hybrid power demand is obtained from Eq. (55)

$Y_{2}(s)=\left(1-R_{\mathrm{c}}\right) \frac{k_{\mathrm{LH}}}{\tau_{\mathrm{s}} s+1} P_{\mathrm{LH}}(s)$
The plant parameters $R_{\mathrm{c}}, k_{\mathrm{LH}}, \tau_{\mathrm{s}}$ for the chosen scenario are

$k_{\mathrm{LH}}=0.06 \times 10^{-6} \mathrm{~W}^{-1}$

$R_{\mathrm{c}}=0.03$

$\tau_{\mathrm{s}}=0.5 \mathrm{~s}$

The design specifications are the following:

(1) A response time of $\tau_{\mathrm{cl}}=0.25 \mathrm{~s}$ is desired for the closed loop system, corresponding to a settling time of approximately $1 \mathrm{~s}$; (2) no steady state error to a step-wise reference input is allowed; and (3) the second current moment overshoot must not exceed $5 \%$ of the requested value.

As the $Y_{2}$ model is a first order system, a PI controller is the minimum requirement to eliminate steady state error to a step wise input. Using the internal model control (IMC) design methodology [29], the parameters of a PI controller can be obtained as a function of the plant parameters $k_{\mathrm{LH}}, \tau_{\mathrm{s}}$ and the desired response time of the closed loop system $\tau_{\mathrm{cl}}$ 

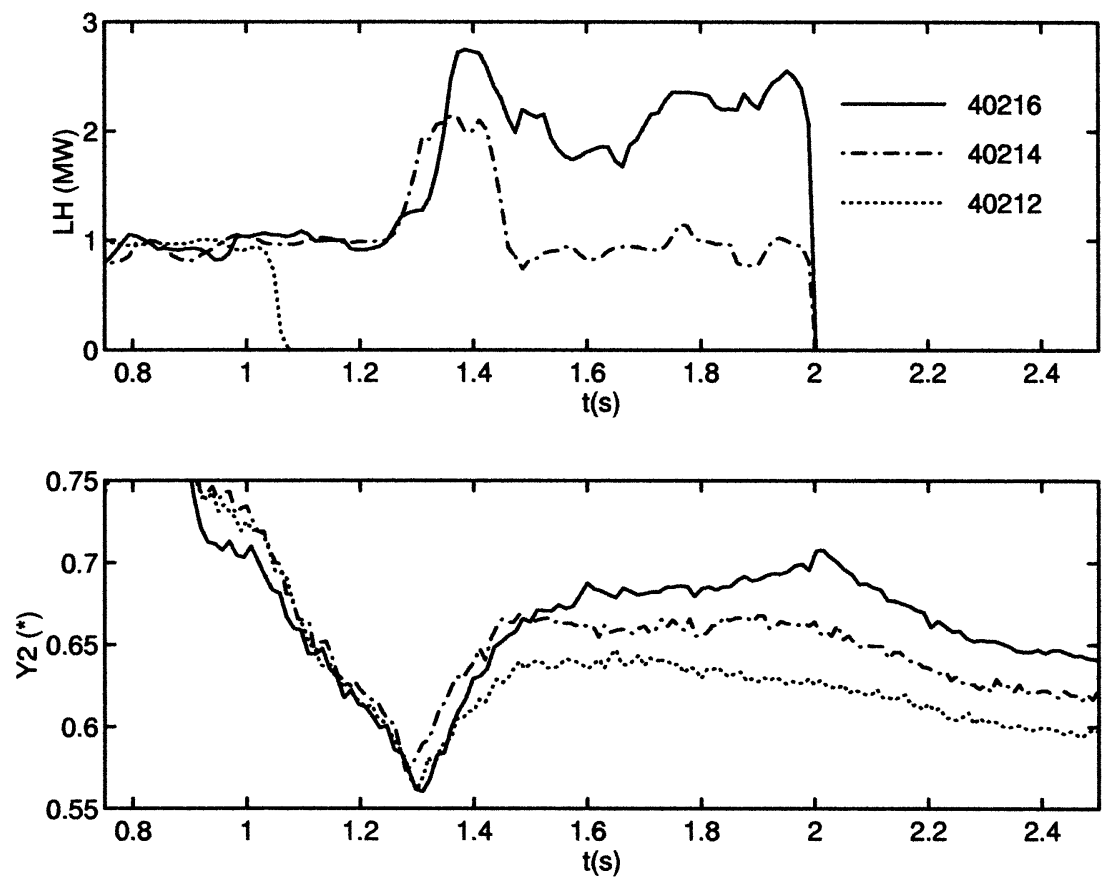

Fig. 16. Current rise phase of three consecutive shear optimisation discharges. Top: Lower hybrid power. Bottom: Second current moment $Y_{2}$. Increasingly higher values of $Y_{2}$ correspond with a progressive broadening of the plasma current profile.

$T_{\mathrm{i}}=\tau_{\mathrm{s}}$

$k_{\mathrm{p}}=\frac{1}{\left(1-R_{\mathrm{c}}\right) k_{\mathrm{LH}}} \frac{\tau_{\mathrm{s}}}{\tau_{\mathrm{cl}}}$

For the plant parameters (Eq. 59) and $\tau_{\mathrm{cl}}=0.5 \mathrm{~s}$, this gives

$T_{\mathrm{i}}=0.5 \mathrm{~s}$,

$k_{\mathrm{p}}=33 \times 10^{6} \mathrm{~W}$

\subsection{Second current moment control experiments}

As discussed earlier, the second moment of the plasma current distribution contains information regarding the off-axis current profile distribution, and feedback control of the second current moment can be used for off-axis current regulation in shear optimization experiments.

The second current moment feedback control experiment is shown in Fig. 15. The aim of the experiment was to broaden the current profile at an early stage of the discharge. To achieve this, 1 MW of lower hybrid power was used in the initial phase to provide central heating and to slow down the current diffusion during the ramp-up. The feedback starts at $t=1.2 \mathrm{~s}, \sim 100 \mathrm{~ms}$ before the $x$-point formation. After the $x$-point formation at $t \cong 1.3 \mathrm{~s}$, the lower hybrid deposition shifts off-axis, providing off-axis current drive/heating. This helps to broaden the profile further, and at $t=2 \mathrm{~s}$ the second current moment set point for feedback (0.7) is reached. The end of the LH pulse was also scheduled at $t=2 \mathrm{~s}$ to avoid a possible plasma disruption triggered by an excess of $\mathrm{LH}$ current drive in the off-axis region.

Three consecutive discharges with increasingly higher values of the second current moment are shown in Fig. 16. These correspond with a progressive broadening of the plasma current profile during the current rise phase. The corresponding deuterium-deuterium nuclear reaction rates $\left(R_{\mathrm{dd}}\right)$ achieved during the neutral beam (NB) heating phase are shown in Fig. 17. These experiments have shown that there is a strong correlation between the second current moment values just after the LH pulse and the maximum reaction 

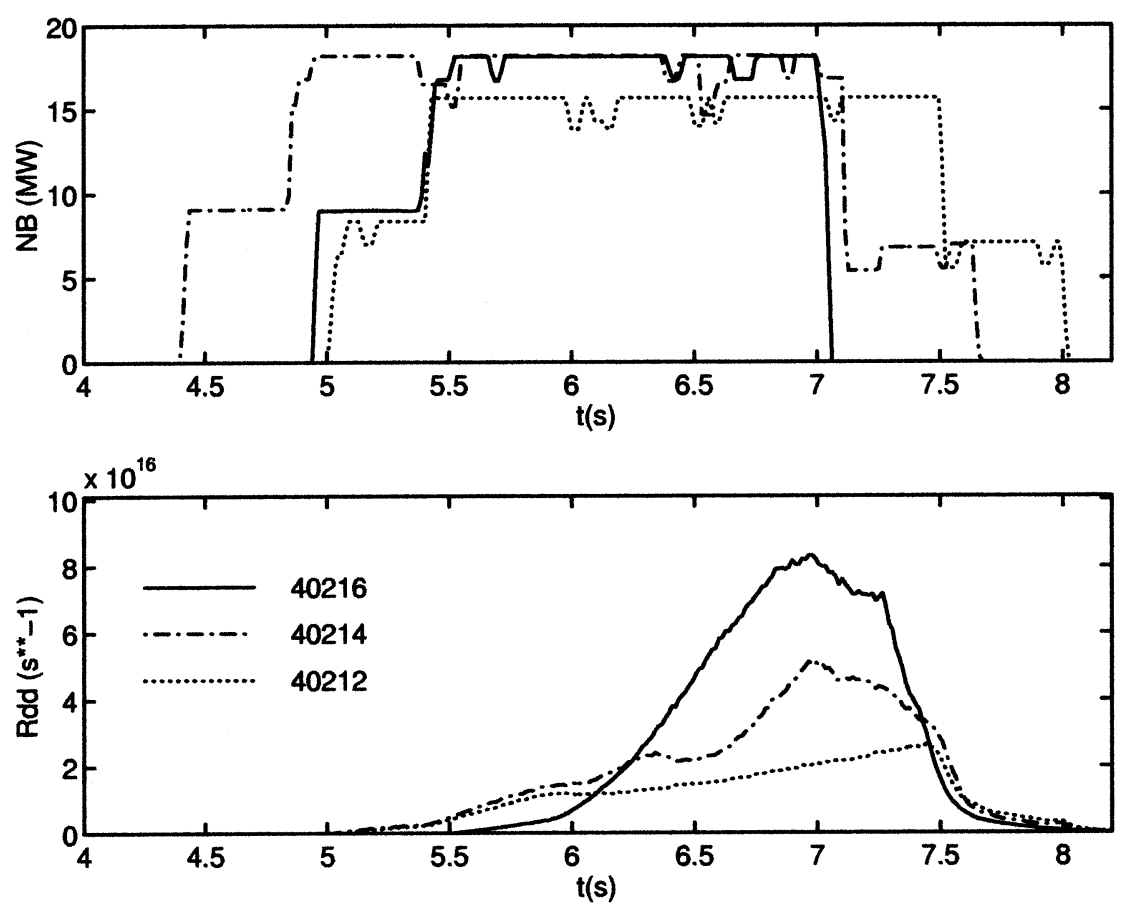

Fig. 17. High power heating phase of three shear optimisation discharges. Top: Neutral beam power. Bottom: Neutron yield $R_{\mathrm{dd}}$. The maximum $R_{\mathrm{dd}}$ values during the NB heating phase are correlated with the $Y_{2}$ values just after the LH pulse (Fig. 16).

rates $R_{\mathrm{dd}}$ achieved during the high power $\mathrm{NB}$ heating phase. The discharge (40216) has a reaction rate $R_{\mathrm{dd}}$ that is $60 \%$ higher than the preceding one (40214), as shown in Fig. 17. This increment can not be explained by a higher level of NB power as the NB power for both shots is roughly the same. The increment in $R_{\mathrm{dd}}$ is the result of an improvement of the energy confinement in the plasma induced by the broadening of the current profile during the current rise phase. This is a general result observed during shear optimization discharges with lower hybrid at JET.

\section{Conclusions}

A current profile control system has been developed and tested at JET. Two separate control loops have been implemented for the LHCD system, non-inductive current control and off-axis current control.
For current profile control at JET, only magnetic diagnostics are available. From these, the loop voltage has been selected for non-inductive current control and the normalised second current moment has been selected for off-axis current control. These provide relatively simple measurements and calculations suitable for real time operation.

A Poynting's theorem energy balance of the Tokamak has been performed and exact expressions for the Tokamak circuit elements $R, L-$ $M, \hat{I}$ have been obtained in a way that is consistent with loop voltage measurements.

When the evolution of $R, L-M, \hat{I}$ in time is related to lower hybrid power input, the loop voltage is best described by a state space model in which the internal states are related to these circuit elements. Based on this, a state space model of a Tokamak for loop voltage control purposes has been presented and validated against JET data. Similarly, a transfer function model between the lower hybrid coupled power and the nor- 
malised second current moment has been obtained. These models have proved to be very useful for the design of the loop voltage and second current moment control systems.

The first experimental results with the system have been presented. Loop voltage has been controlled at $66 \%$ reduction in $2.5 \mathrm{MA}$ discharges. Second current moment control has been used in the initial current raise phase of shear optimization experiments to broaden the current profile, and has contributed to a $60 \%$ improvement of the fusion yield performance. This illustrates the potential benefits of current profile feedback control systems for future devices such as ITER [30] where confinement and stability are crucial.

\section{Acknowledgements}

This paper is part of the work carried out by the first author in fulfillment of the requirements for obtaining a Doctorate degree (Universidad Nacional de Educacion a Distancia, Madrid, Spain). The work described in this paper has been performed in the framework of an European Commission research training program.

\section{References}

[1] JET-Team, Design, construction, and first operational experience with the Joint European Torus (JET). Fusion Technol. 11 (1) (1987) 13-281.

[2] N.J. Fish, Theory of current drive in plasmas, Reviews of Modern Physics, January 1987, pp. 175-234.

[3] M. Pain, H. Brinkschulte, G. Bosia, M. Brusati, J.A. Dobbing, A. Ekedahl, M. Gammelin, C. Gormezano, C. Idelon, J. Jacquinot, Gl Tessop, A. Kaye, M. Lenholm, J. Plancoulaine, Ph. Schild, A. Sibley, T. Wade, C. Walker, R. Walton, G. Wilson. The 15 MW microwave generator and launcher of the lower hybrid current drive experiment on JET, Proc. IEEE 13th Symposium on Fusion Engineering, Knoxville 2, 1989, pp. 1083-1088.

[4] J.M. Maciejowsky, Multivariable feedback design. Addison-Wesley, Reading, MA, 1989.

[5] L. Ljung, System Identification, Prentice-Hall, Englewood Cliffs, NJ, 1987.

[6] N.H. Zornig, H.E.O. Brelen, A. Browne, M.L. Browne, T. Dobbing, C. Gormezano, J. How, F.A. Jesen, T.T. Jones, F.B. Marcus, Q.A. King, F. Rimini, J.A.
Romero, A.G.H. Sibley, F. Söldner, B.J.D. Tubbing. Experimental results using the JET Real Time Power Control system, Proceedings 19th Symposium on Fusion Technology (SOFT-19), Lisboa, Portugal, September 1996.

[7] B.W. Rice, D.G. Nilson, Wroblewski, Motional Stark effect upgrades on DIII-D. Rev. Sci Instrum. 66 (1995) $373-375$.

[8] N.C. Hawkes, Design Study of a Motional Stark Effect Diagnostic for JET. JET-R (96)10.

[9] D.P. O'Brien, J.J. Ellis, J. Lingertat, Local expansion method for fast plasma boundary identification in JET, Nucl. Fusion 33 (3) (1993) 467-474.

[10] L. Spitzer, Physics of fully ionized gases. Interscience Publishers, 1956.

[11] S.P. Hirsman, R.J. Hawryluk, B. Birge, Neoclasical conductivity of a Tokamak plasma, Nucl. Fusion 17 (3) (1977) 611-614.

[12] N.J. Fisch, Conductivity of RF-heated plasma, Phys. Fluids 28 (1) (1985) 245-247.

[13] H. Akaike, Information theory and an extension of the maximum likelihood principle. Proc. 2nd Int. Symp. Information Theory Supp. to Problems of Control and Information Theory, 1972, pp. 267-281.

[14] J.A. Romero, Análisis, modelado y utilización de diagnósticos magnéticos en el control del perfil de corriente en el Tokamak JET, PhD thesis, Universidad Nacional de Educación a Distancia (UNED), Madrid, Spain, November 1997.

[15] C. Litwin, Current diffusion and toroidal electric field response to a non-ohmic current drive, Phys. Plasmas 2 (12) (1995) 4542-4550.

[16] M. Huart, Modelling and computer simulation of large flywheel-generator-diode convertor for fusion experiment pulse load, Proc. 10th Symposium on Fusion Technology, Padova, Italy, September 1978, pp. 377-382.

[17] A. Ekedahl, Y. Baranov, J. Dobbing, B. Fisher, C. Gormezano, M. Lennholm, V. Pericolini-Ridolfini, F. Rimini, J.A. Romero, P. Shild, F. Söldner. Profile Control in JET with Off-Axis Lower Hybrid Current Drive, Proc. 23rd EPS conference on Plasma Physics and Controlled Fusion, Kiev, Ukraine, 24-28 June, 1996.

[18] H. Altmann, E.B. Deksnis, J. Fanthome, C. Froger, C. Lowry, R. Mohanti, M. Nilsen, A. Peacock, M.A. Pick, D. Spencer, R.B. Tivey, G. Vlases. Design of the MKII Divertor with large carbon-fibre composite (CFC) tiles, Proc. 18th Symposium on Fusion Technology, Karlsruhe, Germany, August 1994, Vol. 1 pp. 275-278.

[19] V.V. Parail, O.P. Pogutse, Diffusion of runaway electrons in a Tokamak, Nucl. Fusion 18 (10) (1978) $1357-$ 1362.

[20] M. Lennholm, Y. Baranov, J.A. Dobbing, A. Ekendahl, P. Finburg, B. Fischer, C. Gormezano, C. Gowers, A. Kaye, J. Plancoulanie, F.G. Rimmi, J.A. Romero, P. Schild, A. Sips, F. Smits, F.X, Söldner. Operation of the 3.7 GHz LHCD System in JET, 16th Symposium of Fusion Engineering (SOFE), Champaign, USA, 1995. 
[21] K.Ogata, Discrete Time Control Systems. Prentice-Hall, Englewood Cliffs, NJ, 1987.

[22] B.W. Rice, Demonstration of high performance negative central magnetic shear discharges in the DIII-D Tokamak, Phys. Plasmas 3 (5) (1996) 1983-1991.

[23] The JET Team, Optimisation of JET plasmas with current profile control, JET contributions to the 16th IAEA conference, Montreal, Canada, October 1996.

[24] E.J. Strait, Enhanced Confinement and Stability in DIIID Discharges with Reversed Magnetic Shear, Phys. Rev. Lett. 75 (24) (1995) 4421-4424.

[25] L.E. Zakharov, V.D. Shafranov, Equilibrium of a toroidal plasma with non circular cross-section. Sov. Phys. Tech. Phys. 18 (2) (1973) 151-155.
[26] J. Ellis, The rapid determination of plasma equilibrium parameters at JET from external magnetic measurements, IAEA Technical Committe Meeting on Magnetica Diagnosticos for fusion plasmas, Kharkov, Ukranine, October 1994.

[27] A.J. Wooton, W.A. Copper, $\beta_{\mathrm{p}}$ Analysis for Tokamak Plasma With Anisotropic Pressure and Mass Flow, Plasma Phys. 24 (1982) 1183-1185.

[28] V.D. Shafranov, Determination of the parameters, $\beta$ and $l_{\mathrm{i}}$ in a Tokamak for arbitrary shape of plasma pinch cross section, Plasma Phys. 13 (1970) 757-762.

[29] M. Morari, Robust Process Control, Prentice-Hall, Englewood Cliffs, NJ, 1989.

[30] P.H.. Rebut, ITER: The first experimental fusion reactor, Fusion Eng. Des. 30 (1995) 85-118. 